\title{
Die Wasserversorgung an Bord
}

\author{
Eine medizinhistorische Studie* \\ Von H. SchadewaldT
}

Nächst dem Skorbut ist das Problem der Süßwasserversorgung in vergangenen Jahrhunderten von folgenschwerster Bedeutung für die Schiffahrt gewesen. Standen an Land Hungerkatastrophen im Vordergrund, so war für den Seemann der Mangel an brauchbarem Trinkwasser die alimentäre Notsituation $\varkappa \alpha \tau^{3} \dot{\varepsilon} \xi o \chi \dot{\eta}^{\nu} .1800$ z. B. betonte ein deutscher Autor, Friedrich Henning, der ein vielverbreitetes Diätetisch-medizinisches Handbuch für Seeleute herausgab, ausdrücklich: «... gerade der größte Teil der auf langen Seereisen entstehenden Krankheiten ist dem Mangel oder der schlechten Beschaffenheit der Getränke zuzuschreiben » (78, p. 53).

Verständlicherweise haben sich fast alle Autoren, die die Geschichte der Schiffsmedizin behandelten, auch in mehr oder weniger langen Ausführungen mit der Wasserversorgung an Bord als einem der Kardinalprobleme der Schiffshygiene beschäftigt. Allerdings haben nur wenige es bisher unternommen, die historische Entwicklung der Trinkwasserversorgung zusammenhängend darzustellen (154, p. 26 ff.; 28, p. 21 ff., 54; siehe auch 161, p. 2525 f.). In einer im Entstehen begriffenen Dissertation wird Eike SparWALD das Thema aufgrund bisher zum Teil noch nicht berücksichtigter Quellen ebenfalls behandeln. Auf diese Arbeit sei der interessierte Leser schon jetzt hingewiesen.

Die schmerzlichen Erfahrungen des letzten Weltkrieges haben die Wichtigkeit dieses Problems noch einmal besonders deutlich werden lassen. Bei allen Beteiligten und Experten herrscht seither Übereinstimmung darüber, daß der Tod in Seenot in erster Linie ein Kältetod, in zweiter Linie ein Verdurstungstod und in dritter Linie ein Schocktod ist, daß jedoch kaum einmal ein Schiffbrüchiger im Zweiten Weltkrieg Hungers gestorben war $(18,37,38,115,160,175)$. Soviel Klagen man in den vergangenen Jahrhunderten über die schlechte und unzureichende Ernährung an Bord auch vernehmen konnte, immer stand die Sorge um die Erhaltung des Trinkwassers und seiner Qualität im Vordergrund des Interesses $(160,175$, siehe auch 45 , 169, 170, 171). Die Schwierigkeiten der Trinkwasserversorgung, die man mit

* Erweiterte Fassung eines auf der Henry-E.-Sigerist-Konferenz in Murten 1962 gehaltenen Vortrags. 
der Einführung der Dampfschiffe und der dadurch wesentlich erleichterten Destillation von Seewasser schon überwunden glaubte, haben im übrigen einen neuen Aspekt durch die vielfältigen Diskussionen um Wert und Unwert des Seewassertrinkens durch Schiffbrüchige in Rettungsbooten und auf Rettungsflößen gefunden, die bis heute die Fachliteratur beherrschen $(10,37,38,40,115,160,175)$.

Seeleuten und Schiffsärzten früherer Epochen muß es bereits sehr klar gewesen sein, daß das Trinken von Seewasser keine Rettung vor dem Verdurstungstode brachte, und Seewassergenuß ist auch stets nur als Therapeutikum in kleinen Dosen $\left(102,2^{\circ}\right.$, p. 60$)$, sei es zur Bekämpfung der Seekrankheit $(14 a, 74,79 a, 178)$, sei es als Antiskorbutikum $(91,92,155,172)$ empfohlen worden. Die Versuche Peters des Grossen (1672-1725), die Kinder seiner Matrosen durch ständige Aufnahme von kleinen Mengen Meerwasser an das Seewassertrinken zu gewöhnen, dürften keinen diesbezüglichen Erfolg gebracht haben (154, p. 28; 103, p. $323 \mathrm{ff}$.). Trotz des heroischen Selbstversuchs von Alain Bombard, des französischen Ozeanologen, der 1952 ohne Trinkwasservorräte in einem Segelschlauchboot den Ozean überquerte und seinen Wasserbedarf durch fraktioniertes Seewassertrinken und das Aussaugen der Lymphe von gefangenen Fischen deckte (24), sind sich die Fachleute darüber einig, daß Seewassergenuß spätestens am 7. oder 8. Tag zum unweigerlichen Ende des Betreffenden infolge mit dem Leben unvereinbarer osmotischer Mineralverschiebungen im Organismus führen muß, wofür englische Autoren eindeutige Mortalitätsziffern aus dem letzten Weltkrieg anführten $(37,46)$. In einer Gruppe von Schiffbrüchigen, die Seewasser tranken, betrug die Mortalität 38,8\%, in einer anderen, die darauf standhaft verzichtete, nur 3,3\% (115). Im übrigen konnte kürzlich der deutsche Ernährungsphysiologe HaNs Glatzel (geb. 1902) nachweisen, daß für die Eliminierung der in $500 \mathrm{ml}$ Seewasser enthaltenen Salze bei einem hungernden und durstenden Menschen $700 \mathrm{ml} \mathrm{Süßwasser} \mathrm{benötigt} \mathrm{werden,} \mathrm{d.h.}$ daß außer den $500 \mathrm{ml}$ des aufgenommenen Wassers der bereits ausgedörrte Organismus noch $200 \mathrm{ml}$ aus seinen Beständen zur Ausschwemmung vornehmlich von $\mathrm{Na}$ und $\mathrm{Cl}$ zur Verfügung stellen muß (64). Die optimistischen Angaben von Franz VolHard (1872-1950), die allein aufgrund von Freiwilligenversuchen in der Klinik gewonnen wurden, konnten von der Schiffsmedizin nicht bestätigt werden. Aber auch VolHaRD errechnete sogar, daß für $500 \mathrm{ml}$ Seewasser etwa $400 \mathrm{ml}$ zusätzliches Körperwasser benötigt werden, wobei allerdings nach seiner Auffassung bei einer wasserarmen Kost von 1500 Kalorien erst nach 10 Tagen mit ernsthaften Störungen zu rechnen 
wäre (179). Der berühmte Klageruf des englischen Dichters SAmuel Taylor Coleridge (1762-1834): "Water, water everywhere, nor any drop to drink" (35) oder der Vergleich der Durstqualen der Seeleute mit den Leiden des Tantalus: «... imiter Tantalus mourant ainsi soif au milieu des eaux» (99) haben von der Entdeckung Amerikas an bis heute nichts von ihrer erschütternden Aussagekraft eingebüßt.

Es ist beliebt, in medizinhistorischen Exkursen mit Hippokrates (460-377 v. Chr.) zu beginnen, aber in diesem Fall sind wir tatsächlich be-

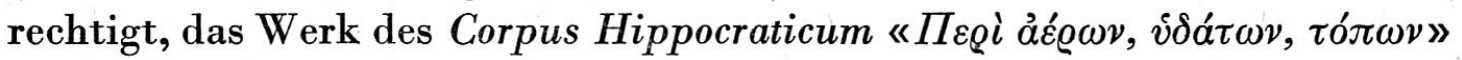
zu zitieren, wo von Kapitel 7 an ausführliche und heute noch beherzigenswerte Ausführungen über Wasserversorgung und geeignetes Trinkwasser zu finden sind (80). Daß stehende Gewässer im Sommer für die Gesundheit gefährlich werden können, ist auch heute noch eine Binsenweisheit. Dieses Wasser war nach Ansicht des Autoren jener Schrift «warm, dick, stinkend,

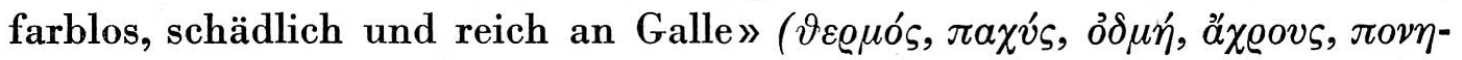

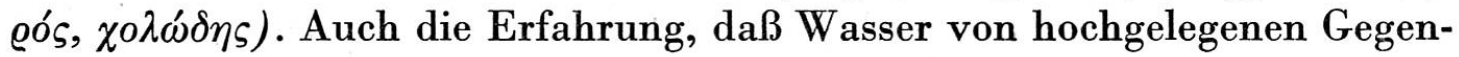
den sowie solches, das als «salzig, unerweichlich und hart» imponierte

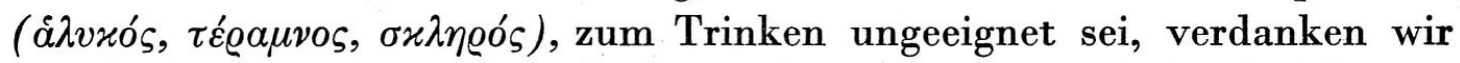
bereits den hippokratischen Autoren, die andererseits wieder das Regenwasser als besonders rein, das Schnee- und Eiswasser dagegen als schädlich betrachteten und damit ein Verdikt über diese wichtige Süßwasserquelle aussprachen, das erst durch die Eiswasserexperimente Kapitän JAMES Соокs (1728-1779) während seiner zweiten Weltreise in die Antarktis 1773 aufgehoben wurden, auf die wir noch zu sprechen kommen werden (siehe S. 78). Nach den Hippokratikern war das aus Schnee und Eis gewonnene Wasser ohne Ausnahme schlecht, das «Klare» würde sublimiert, das «Schwere, Schmutzige» jedoch zurückbleiben. Immerhin hatten sie schon empfohlen, das Regenwasser abzukochen und durchzuseihen, weil es sonst einen schlechten Geruch behalte und sich nach dem Genuß des unpräparierten Wassers eine gewisse Heiserkeit und eine belegte Stimme einstellen könne $(80,54$, p. 15$)$.

Seit dieser Zeit hat die Sorge um sauberes, einwandfreies Trinkwasser die Ärzte aller Epochen nicht wieder verlassen. Plinıus (23-97 n. Chr.) war z.B. der Überzeugung, daß man an bestimmten Stellen mitten im Meer Süßwasser aus tief am Meeresgrund austretenden Quellen gewinnen könne (140, Band 1, Lib. 2, Cap. 227, p. 354): «Sed fontium plurimorum natura miro est fervore ... in mari, ut inter Italiam et Aenariam in Baiano sinu ... Nam dulcis haustus in mari plurimis locis ...», weil das Salzwasser schwerer 
als Süßwasser sei und letzteres daher auf ersterem schwömme (140, Band 1, Lib. 2, Cap. 224, p. 352): «Mirabilius id faciunt aquae dulces iuxta mare ut fistulis emicantes ... Ideo et marinae, quarum natura gravior, magis invecta sustinent.» Diese Ansicht wurde auch in den pseudoaristotelischen «П@o-

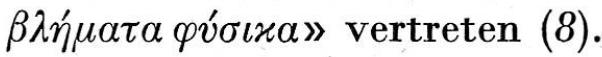

Ganz ähnliche Erfahrungen machte noch 1688 der englische Weltreisende William Dampier (1652-1715), der noch eine Meile vor der Küste von Siam reichlich Süßwasser «wannmehr wir auch alsobald etliche Tonnen damit fülleten» abschöpfen konnte und auch der Ansicht war, daß das Flußwasser noch eine Strecke weit im Meer auf dem schweren Seewasser schwimmen würde (39).

Aristoteles (384-322 v. Chr.) lieferte dann bereits einen direkten Hinweis auf Versuche, aus dem Meerwasser süßes Trinkwasser zu gewinnen. In seiner Meteorologia und in ähnlicher Weise auch in der Tierkunde ist von angeblich «wächsernen Gefäßen » die Rede, die innen hohl waren, mit einem festen Verschluß versehen werden konnten und in die, wenn sie 24: Stunden neben dem Schiff ins Wasser getaucht wurden, eine kleine Menge süßes und trinkbares Wasser einsickern sollte $(6,7,81)$ : «Nam siquis vas caereum effingit, ipsumque demittat in mare ore ita circumquaqua obturato ut maris aqua introire non queat. Quicquid marini liquoris per caerea latera in vas ingreditur potabilem capit saporem » (6).

Alexander von Aphrodisias (um $200 \mathrm{n}$. Chr.), der bekannte AristoTELES-Kommentator, hat diese Hinweise ebenfalls aufgenommen und erwähnte, daß mit Hilfe von Wachsgefäßen aus Meerwasser Süßwasser gewonnen werden könne: «Fidem affert per cuiusdam rei admistionem effici salsum saporem: quod quicquid marini liquoris per caerea vasa iniecta mari ingreditur, exprimitur, et percolatur, ad dulcem reddit naturam» (2).

Dagegen machte der Chemiehistoriker Edmund Oskar von LipPManN (1857-1940) mit Recht darauf aufmerksam, daß, wenn man anstatt $\dot{\alpha} \gamma \gamma \varepsilon i \tilde{o} \nu$

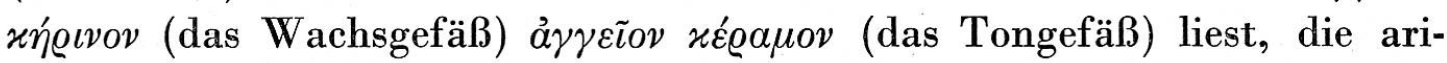
stotelische Angabe durchaus verständlich erscheint und daß es Nachuntersuchern tatsächlich gelang, mit Hilfe von Tongefäßen, die allerdings bereits schon längere Zeit im Wasser gelegen haben mußten, geringe Mengen trinkbaren Wassers zu erhalten. Auf diese Weise konnten immerhin in einem 1 1/4-1-Krug $50 \mathrm{ml}$ einwandfreies Süßwasser gewonnen werden (104).

Die Methode der Seewasserfiltration mit den angeblichen Wachskugeln tauchte in der schiffsmedizinischen Literatur immer wieder auf, allerdings meist ohne Bezugnahme auf den Erstautor. Freilich berichteten die be- 
treffenden Schriftsteller meist nichts über eigene Versuche; sie begnügten sich mit der Bekanntgabe des Gehörten oder Gelesenen, ohne weitere Quellen anzuführen. Versuche, mit Hilfe von entsprechend geformten Wachskugeln Süßwasser zu gewinnen, sind meist nicht geglückt (51, p.479, 84). Im Mittelalter kannte Johannes Anglicus (John of Gaddesden) (1280? bis 1362) vier verschiedene Verfahren der Entsalzung von Seewasser (4, 72, p.X): Einmal das von Aristoteles mit Hilfe von Wachskugeln, «nodo fiat de cera usum», dann die noch zu besprechende Destillation mittels Alembik, «Distillatur aqua salsa per alembicum suaviter et erit aqua dulcis», weiter die Filtration durch Sand und schließlich das Auffangen von Trinkwasser in Leinentücher, die über ein erhitztes Wassergefäß gehängt wurden, ähnlich dem Vorgehen des heiligen BAsilius (um 330-379) (siehe S. 52) $(4,72$, p. X) : «bulliat aqua salsa in cacabo et linteamen mundim ponatur super fumum, postea exprimatur et transeat erit illa aqua dulcis.»

1580 berichtete der 1556 nach Brasilien segelnde Reisende JEAN DE LERY über diese Methode: «...il ne seroit pas possible en ceste extremité de boire... je responds que quelque recepte qu'on ne peust alleguer de la faire passer par dedans de la cire, ou autrement d'allambiquer... » (99). Stephen Hales (1677-1761), der ideenreiche englische Geistliche, dem wir noch begegnen werden, behauptete dann 1739, daß mit Hilfe von Wachskugeln nur sehr kleine Mengen von Süßwasser, wenn überhaupt, gewonnen werden könnten, daß aber die schnell verschmutzten Kugeln erst wieder in Süßwasser gereinigt werden müßten; auf hoher See bei Trinkwassermangel eine fast unmögliche Forderung $(72$, p. XI): "But this is only a matter of curiosity, because but a very small Quantity can be thus prepared; and in order to make those waxen Bowls fit for farther Filtration, they must be cleansed from the Salt, by being washed in fresh Water."

Johann Friedrich Wilhelm Otto referierte noch 1792 über Versuche, mit Hilfe von hohlen Wachskugeln aus Salzwasser Süßwasser zu gewinnen, was aber wegen starker Verschmutzung der Kugeln angeblich nur jeweils einmal gelang. Erst nach vielen Stunden begann eine geringe Menge relativ süßen Wassers durchzutreten $(81,131$, p. 80). Eine andere Art der Süßwassergewinnung gab Plinius bekannt, der glaubte, mit Hilfe von Wollvliesen, die rund um das Schiff ins Wasser gehängt wurden, durch natürliches Verdampfen des Salzwassers Süßwasser zurückhalten zu können, ein Verfahren, das sicherlich keinen Erfolg gebracht haben dürfte (51, p. 480, 54 , p. $15,81,97$, p. 56,140$)$. Andererseits waren auf den griechischen Galeeren, die je nach Zahl der Ruderbänke als Trieren, Tetreren oder Penteren 
bezeichnet wurden, wie Athenaios in seinem um 200 n. Chr. entstandenen

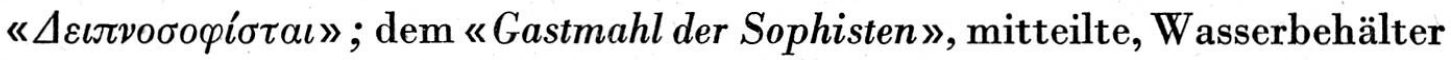
bekannt. Athenaios beschrieb eine Riesentriere des Tyrannen Hieron von Syrakus (gest. 467 v. Chr.), die zuerst «Syrakusia» später «Alexandrias» genannt wurde und erwähnte dabei neben Trinkgefäßen ( in einer sogenannten «Kammer der Aphrodite» auch eine $v \delta \varrho o \vartheta \eta \dot{~} \varkappa \eta$, eine Zisterne, die angeblich $2000 \mu \varepsilon \tau \varrho \eta \tau \alpha \tilde{\iota}$ (ungefähr 74000 Liter!) Wasser hätte aufnehmen können und die aus gepechten Planken bestand und mit geteertem Segeltuch gedeckt wurde (9, V, 206, Band 2, p. 432 ff.; 54, p. 16). Wenn auch die Wassermenge unmäßig übertrieben scheint, so muß es sich doch offensichtlich um ein für eine längere Seereise wohlausgerüstetes Schiff gehandelt haben. Athenaios war es im übrigen auch, der entgegen der Ansicht der Hippokratiker das Schneewasser durchaus als Trinkwasser akzeptierte, weil nach seiner Auffassung die trinkbaren Anteile des Wassers oben schweben würden und, da Eis leichter als Wasser sei, darin der trinkbare Anteil kondensiert sein müßte (9, II, 42, Band 1, p. 182 f.). Im allgemeinen wurde das Wasser an Bord phönizischer und griechischer Schiffe sonst in Leder- oder Ziegenhautschläuchen bzw. in Tonkrügen aufbewahrt (149, p. 101, Ann., p. 231).

Generell wird dem heiligen BASILIUs die erste Erwähnung der Meerwasserdestillation zugeschrieben $(15,20,51$, p. 478, 72, p. X, 97, p. 57, 81, 161, p. 2525). Basilius behauptete in seinen Homilien, einem exegetischen Predigttext im Sinne der späteren Scholien, daß Regen über dem Meer, der ja als Süßwasser niederfällt, dadurch entstünde, daß durch die Verdunstung, die Basilius «Percolatio», «Durchseihung», nannte, das salzige Meerwasser seine «amaritudo », Bitterkeit, verlöre (15): «... impulsum quae fuit a vento illud movente tum superficie disrupta foras erumpit, atque emendante amaritudinem percolatione, potui aptum evadit. » Er kannte aber auch einen künstlichen Weg, den die Seeleute einschlugen, die nämlich das Meerwasser sieden und die Dämpfe in Schwämmen auffangen würden, um in Notfällen Trinkwasser zur Verfügung zu haben $(15,28$, p. 21,51, p. 478 und $480,2^{\circ}$, p. 672,81$)$ : «Quin etiam ipsam maris aquam a navigantibus coneoqui videre licet, qui vapores spongiis excipientes, in necessitatibus utcumque penuriam elevant.»

Schon früher dürfte aber Alexandros von Aphrodisias (um $200 \mathrm{n}$. Chr.) in Athen eine Art Destillation gekannt haben (2, 54, p. 16). Den Hinweis des Polyhistors Drodonus von Sizilien, dessen Historische Bibliothèk die Zeit bis $54 \mathrm{n}$. Chr. behandelte, daß «das Feurige die natürliche Wirkung 
habe, alles Flüssige süß zu machen », kann man dagegen auf keinen Fall als Vorahnung der Destillation betrachten, hatte doch Diodor damit nur erklären wollen, warum das Nilwasser, das nach ihm aus sehr heißen Gegenden kam, besonders süß erschien $\left(43,51\right.$, p. $479,2^{\circ}$, p. 673$)$.

Im ganzen Mittelalter bestand die Sitte, den im Mittelmeer segelnden Schiffen größere Wassermengen in Holzfässern mitzugeben, denn man war inzwischen von der Übung der Griechen abgewichen, sich ausschließlich auf die Küstenschiffahrt während der Sommermonate zu beschränken, wobei natürlich jederzeit die Möglichkeit bestand, frisches Quellwasser aufzunehmen. Wir wissen z. B. von einem Vertrag Ludwigs IX. von Frankreich (1214-1270) mit Marseille aus dem Jahre 1246, wonach jedes Schiff für die Kreuzzugsreisen 2000 «Mezaroles» Wasser fassen sollte (54, p. 16). Auch Avicenna (980-1037) hatte in seinem Lehrgedicht Cantica den Rat erteilt, bei Schiffahrten, vor allem im Winter, viel Wasser in geeigneten Gefäßen und flüssige Nahrung mitzuführen $(11,130)$. Bei den relativ kurzen Seefahrtszeiten - eine Reise von Venedig bis nach Jaffa oder Akkon dauerte im allgemeinen einen bis anderthalb Monate - stellten sich noch keine besonderen Probleme der Wasserkonservierung ein. Immerhin war auch schon in jener Zeit die Ansicht vorherrschend, daß man Wasser mit Essig (54, p. 17) oder, bei wohlhabenderen Passagieren, mit Wein versetzen sollte $(32$, p. 26, 124). Übrigens versorgten sich die Passagiere der ersten Klasse, wenn man so sagen darf, noch zusätzlich mit zahlreichen $\mathbb{W}$ einflaschen, um sich gegen etwaige Notfälle zu sichern (124). Nach wie vor war es aber ein Hauptanliegen der Kapitäne, sich frisches Quellwasser zu verschaffen, insbebesondere solches, das über sandigen Boden gelaufen war. Schon in den hippokratischen Schriften war durch Sand gesickertes Wasser als besonders gesund betrachtet worden $(54$, p. 15, 80). So hatte z.B. der englische Seefahrer Thomas Cavendish (1555?-1592) 1588 den Wert von durch Sand gefiltertem Trinkwasser deutlich erkannt (71, Band 11, p. $293 \mathrm{ff} ., 87$, Band 1, p. 95). Die Beschaffung frischen Quellwassers war aber in unbekannten und unzugänglichen Gegenden, insbesondere in den Tropen, nicht immer einfach. Oft konnte man mit den unhandlichen Holzfässern gar nicht durch das Dickicht der Küste bis zur Quelle vordringen, dann mußten Ketten gebildet werden, die das kostbare $\mathrm{Naß}$ in Holzeimern bis zur Schaluppe weiterreichten. Dort wurde es der Einfachheit halber in das Boot gegossen; die Ruderer saßen also buchstäblich bis zum Rumpf im Wasser (32, p. 28). $\mathrm{Daß}$ diese Transportmethode nicht gerade hygienisch war, ist offensichtlich (54, p. 21). Später verwandte man jedoch Leinwandschläuche, die sich der 
Bootsform anpaßten (54, p. 39). Erst vom 18. Jahrhundert an gab es in den größeren Häfen sogenannte «Wasserboote» (54, p. 38). Die Qualität des von diesen Booten angebotenen, oft gar nicht billigen Trinkwassers ließ aber beim Fehlen jeder obrigkeitlichen Überwachung des öfteren zu wünschen übrig. Die Wassertanks wurden nämlich gewöhnlich durchschnittlich nur alle zwei bis drei Jahre mit Schwefel und Kreide gereinigt (54, p. 38). Als einen Vorläufer dieser Tender kann man vielleicht das englische Lazarettschiff betrachten, das 1702 "may bring a good quantity of Thames water for the fleet" (27, 87, Band 2, p. 240), wobei bezüglich der besonderen «Qualitäten» des Themsewassers auf S. 57 verwiesen sei.

Manche Empfehlungen sollten die Kapitäne vor der Aufnahme ungeeigneten Wassers schützen. Besonders originell war der Vorschlag von GEorg Pictorius (um 1500-1569) 1557, in fremden Ländern die Brunnen voll auszuschöpfen und dann zur Kontrolle der Reinheit ein Tuch hinunterzulassen. Wenn sich kein Moos daran verfing, sollte es ohne Bedenken trinkbar sein $\left(137\right.$, fol. $\left.20^{\mathrm{v}}\right)$.

Im Mittelalter und in der beginnenden Neuzeit dürften die Schiffschirurgen jedoch einen maßgebenden Einfluß auf die Auswahl der Wasserplätze noch nicht gehabt haben $(32$, p. 28,54, p. 36, 136, p. 22). Ihrer Aufsicht wurde das Trinkwasser in der französischen Kriegsmarine offiziell erst 1798 unterstellt: «L'orsque dans les pays étrangers il faudra faire de l'eau, le chirurgien-major se portera sur les lieux pour s'assurer de sa bonté» (54, p. 36).

Bei den Handelsmarinen dürfte dies im allgemeinen schon etwas früher der Fall gewesen sein.

Bereits seit alter Zeit wurde ungesundes Trinkwasser als Ursache verschiedener Erkrankungen, insbesondere von Diarrhöen angesehen, und arabische Autoren rieten z. B. Reisenden an, stets etwas heimatliche Erde mit sich zu führen, um diese in verdächtiges Trinkwasser zu schütten und sich dadurch gegen Durchfallsgefahren zu feien $(2 a, 48,168 a)$. Bei der Lektüre von zeitgenössischen Berichten fällt aber auf, daß Durchfallsepidemien sich fast stets während oder kurz nach dem Besuch von Häfen einstellten, praktisch aber kaum jemals auf hoher See auftraten, obwohl gerade dort das verfaulte und widerliche Wasser, das oft genug wegen seines Geschmacks und seines Aussehens beanstandet wurde, genossen werden mußte. Dieser interessanten Frage soll in einer gesonderten Untersuchung noch näher nachgegangen werden. Hier sei nur ein Bericht für viele zitiert : «Es ist außer allem Zweifel, daß diese Krankheiten (Ruhr und Diarrhöen) von der Feuchtigkeit des 
Wetters und den Ausdünstungen des Landes veranlaßt werden, denn sobald wir wieder in die freye See und so weit hinaus kamen, daß die schädlichen Ausdünstungen vom sumatrischen Ufer uns nicht erreichen konnten und das Schiff hinlänglich gereinigt war, so wurde die Krankheit milder und der ruhrhaften Ansteckung Grenzen gesetzt ...» (33). Und ein anderer Autor betonte: «Es gibt viele, die sich mehr vor dem Mangel als vor dem Verderben des Wassers fürchten» (173).

Mit der Entdeckung Amerikas 1492 durch Christoph Kolumbus (1451-1506) und des Seeweges nach Indien 1498 durch VASco DA GaMA (1469-1524) traten allerdings an die Seefahrt - auch auf dem Gebiet der Trinkwasserversorgung - völlig neue Probleme heran. Es kam nicht nur zum Auftreten der bekannten Avitaminosen wie Skorbut, Beriberi und der Xerophthalmie, es kam nicht nur zu Versorgungsschwierigkeiten mit den landläufigen Lebensmitteln, sondern in erster Linie zu akuten Wassermangelzuständen. Wurde zu Ende des letzten Jahrhunderts eine Frischwassermenge von 6 bis 7 Liter als tägliches Mindestmaß für notwendig erachtet, (127, p. 227, 149, p. 234) - in der französischen Marine waren Anfang dieses Jahrhunderts 2 Liter Trinkwasser und 10 Liter süßen Waschwassers üblich (54, p. 72) und W. Riegel gab 1914 für die deutsche Marine 8 Liter Trinkwasser und 12 Liter Waschwasser als Normalsüßwasserverbrauch an (147) - so betrug die Süßwassermenge, die auf den in den sogenannten Kalmen segelnden Schiffen, die oft mit tage- und wochenlangen Flauten zu rechnen hatten, pro Kopf der Besatzung ausgeteilt werden konnten, manchmal nur $1 / 2$ bis 1 Liter pro Tag (87, Band 1, p. 98, 149, p. 170, Ann. p. 243) und erreichten das heute für die Aufrechterhaltung der Gesundheit geforderte und gerade noch zulässige Minimum von 1,5 Liter Trinkwasser nicht mehr. An Waschen des Körpers und der Kleidung, Ausspülen des Mundes und an das Kochen von Speisen in Süßwasser war überhaupt nicht mehr zu denken. Salzwasser löst bekanntlich Seife sehr schlecht und ist für die Reinigung der Wäsche vollständig ungeeignet. Die Körperwäsche mit Salzwasser führt nicht selten zu Hautreizungen, insbesondere in den Tropen, wo die Transpiration erheblich verstärkt ist.

Die wechselnden Segelzeiten - eine Reise nach dem Senegal konnte zwei bis sechs Monate, nach dem Kap der Guten Hoffnung drei bis zehn, nach Ostindien sechs bis achtzehn Monate dauern, eine Reise nach Westindien konnte im besten Fall in drei bis vier Wochen, im schlechtesten Falle in zwanzig Wochen zum Ziel führen, wenn man nicht Schiffbruch erlitt - und die unerhört hohen Besatzungsstärken warfen natürlich für die Sicherung 
der Trinkwasserversorgung neue schwierige Probleme auf. Man erkannte bald, daß das Trinkwasser in frischen Holzfässern eher faulte als in alten, abgelagerten (16, p. 25, 54, p. 27) und daß Quellwasser, das als besonders rein galt - ein paradoxes Phänomen -, eher zu krankhaften Störungen führen konnte als das an sich schon schmutzige Themsewasser $(26 a, 27,72$, p. 58, 87, Band 2, 240). Es bestand damals im übrigen die Auffassung, daß Trinkwasser an Bord, wenn es nicht innerhalb der ersten drei bis vier Wochen während der Seereise verbraucht werden konnte, erst zwei- bis dreimal faulen müßte $(28$, p. 28,30 , p. 62,32 , p. 24,54 , p. $29,100,149$, p. 103 , Ann., p. 232, 131, p. 77, 93, 95) und dann erst, also oft nach zwei bis drei Monaten trinkbar würde, weil dann die trübe, gelbliche, ekelhaft nach Schwefel stinkende Brühe durch Absetzen der Schwebestoffe wieder zu leidlich klarem Trinkwasser geworden war: «(L'eau devient) excellente et aussi légère peut-être que l'eau distillée parce que toutes les matières hétérogènes se sont précipitées et restent en sédiment au fond des barriques » (95).

War man lange Zeit der Auffassung, daß diese Fäulnis als typische Begleiterscheinung der Seefahrt aufträte, insbesondere bei ständigem Stampfen und Schlingern, und bestimmten, der See eigentümlichen meteorologischen Verhältnissen, so betonte 1767 AntoIne PoIssonnier-Desperrières (1722-1793), daß diese putride Zersetzung die Folge der Auflösung im Wasser befindlicher «animalcula» wäre, deren «volatile Teile» sich am Boden des Fasses absetzen würden (141). Damit war eine tatsächliche existierende Noxe vermutet worden, ohne daß aus diesen Äußerungen allerdings schon auf eine Vorahnung der Bakteriologie geschlossen werden dürfte, da Poissonnier-Desperrières sicherlich die makroskopisch erkennbaren, im Wasser befindlichen Insekten oder das Plankton damit gemeint haben dürfte (54, p. 29). 1875 wurde dann von französischen Hygienikern beobachtet, daß sich tatsächlich in jahrelang gelagertem Trinkwasser massenhaft Algen entwickelt hatten, vor allem Oscillaria viridis und Palmella, die schließlich etwa einen Drittel des Inhalts der Behälter füllten. Dieses Wasser wurde im übrigen besser beurteilt als solches, das nur kurz gelagert hatte. Diese merkwürdige Tatsache gab damals zum Erstaunen Anlaß, ließ aber die Berichte der alten Seefahrer, die bisher zum Teil als «Seemansgarn» abgetan worden waren, in einem andern Lichte erscheinen $(61$, p. 33 f.): «La présence de ces algues prouve que les eaux dêgout sont améliorées depuis qu'elles ont été recueillies. Ce fait semble d'abord paradoxale, mais il l'ai vérifié plusieurs fois.» Und: «Les marins ont souvent 
signalé un fait semblable, dans l'eau embarquée pour l'alimentation. Cette eau, conservée dans des fûts, se gâte d'abord, puis elle redevient potable.» Algen sollten im übrigen Nitrite, Sulfite und Sulfate im Wasser verbrauchen und auf diese Weise zur Selbstreinigung des Trinkwassers beitragen können (81). Hales behauptete schon 1739, daß: “putrid Water, tho' nauseous, is not observed to be hurtful to human Bodies" (72, p. 58). Freilich war sein Verfahren des Zusetzens von Mineralien oder Metallsalzen in kleinsten, auch oligopharmakologisch wohl kaum wirksamen Dosen vom heutigen Standpunkt weniger zu empfehlen als das von ihm ebenfalls zitierte Vorgehen von Hermann BoerhaAve (1668-1738), der durch Abkochen alle Lebewesen im Wasser zu vernichten hoffte $(23 a, 72$, p. 58).

Sogar der bewußte Zusatz von Algen und anderen Grünpflanzen, wie dem legendären Sargasso-Gras, zur Gewinnung einwandfreien Frischwassers ist schon 1684 von MaRTin Lister (1638-1712) erwogen worden (105): "From these Plants did distil dayly (tho' in a small quantity) a fresh, very sweet, and potable water, which hath no Empegreuma, or unpleasant tast, as all those distilled by fire necessarily have. I urge this experiment, as the most naturel, most easy, and most safeway of having sweet water from the sea." Das Faulen des Wassers wurde z.B. von Johann Georg LeutMANN (1667-1736), Theologe und Mathematiker, später Professorin St.Petersburg, 1697 zur Reinigung von bereits destilliertem Wasser benutzt, in dem er diesem Hausenblase, eine heute noch wegen seiner Klärfähigkeit beim «Schönen des Wein» gelegentlich verwandte Innenhaut der Schwimmblase bestimmter Fische, zusetzte $(81,100,131$, p. 80, siehe auch 72, p. 58). Dieses Phänomen ist heute durchaus erklärlich, wissen wir doch, daß die Pollution des Wassers, insbesondere in Verbindung mit dem noch zur Fäulnis neigenden eiweißhaltigen frischen Holz neuer Fässer, zuerst zu einem Wuchern von Bakterien und Algen Anlaß gibt, daß dieser Zustand aber bei einem Überhandnehmen der letzteren wiederum zu einer Einschränkung des Bakterienwachstums führt und daß sich die Algen dann schließlich am Boden des Gefäßes absetzen (149, p. 232) und so nach einer gewissen Zeit ein relativ klares und einigermaßen genießbares Wasser durchaus entstehen kann $(127$, p. 226). Bereits infiziertes und mit organischen Substanzen angereichertes Wasser fault natürlich schneller, macht aber dadurch auch eher die notwendigen Reinigungsprozesse durch.

Daraus ergab sich die merkwürdige Tatsache, daß manche englischen Kapitäne lieber Themsewasser mitführten, weil diese besonders leicht verschmutzbare Trinkwasserquelle schneller faulen und dann eher für den 
Wassergenuß zur Verfügung stehen sollte $(27,87$, Band 2, p. 240, 129). Kein Geringerer als Sir George Anson (1697-1767) hatte diese Fähigkeit des Themsewassers, schnell zu faulen und dafür aber auch bald wieder als einwandfreies Trinkwasser zur Verfügung stehen, in einem Vergleich mit ähnlich beschaffenem Süßwasser auf der Insel Ste-Catherine, nahe der «Papageieninsel» vor der brasilianischen Küste, lobend erwähnt (5, p. 38): "L'eau est admirable, et se conserve sur mer aussi bien que celle de la Thamise. Car après avoir été un ou deux jours en barriques, elle commence à travailler avec une puanteur insuppportable, et se couvre d'abord d'une écume verdâtre; mais peu de jours après cette écume va à fond, et l'eau devient parfaitement douce, et claire comme du cristal.»

Freilich war das nicht immer der Fall, und die «Selbstreinigung» trat nicht jedesmal mit gewünschter Präzision ein. Zum Beispiel wurde das Themsewasser 1788 schon viele Monate vor der Abfahrt an Bord genommen und zeigte sich dann beim Aufschlagen der Fässer so, wie es der englische Schiffsarzt Leonard Gillespie (1758-1842) beschrieb (86, 106, p. 91): "Thames water filled six month ago at Deptford in unseasoned casks, which were now filled with a dilute sort of ink-putrefaction has made it fetid and stinking."

So ist vielleicht auch die Übung zu verstehen, zuerst die Biervorräte auszugeben und erst dann die Trinkwasserfässer aufzuschlagen (19, p. 20, 87, Band 2, p. 184, 156, 187): «Das Tranck belangend, wann man ausfahret, gibt man so lang Bier, als es wäret. Wann solches auf ist, bekommt man einen gantzen Tag mehr nicht als ein Maß Wasser, wenns noch reichlich hergeht» (156).

Am abstoßendsten und ekelhaftesten war also wohl das Trinkwasser zwischen der dritten Woche und dem zweiten Monat, und ein solches Wasser muß der deutsche Dichter Johann Gottrried Seume (1763-1810) aus Not, wie viele andere freiwillige und unfreiwillige Passagiere jener Zeit, nolens volens getrunken haben, der, von hessischen Häschern eingefangen und gezwungenermaßen als Soldat nach Amerika verfrachtet, während der Überfahrt seine Erfahrungen mit dem Trinkwasser an Bord folgendermaßen beschrieb $(54$, p. 28, 154, p. 28, 167): «Das schwer geschwefelte Wasser lag in tiefer Verderbnis. Wenn ein $\mathrm{Fa}$ heraufgehoben und aufgeschlagen wurde, roch es auf dem Verdeck wie Styx, Phlegeton und Kokytos zusammen. Große fingerlange Fasern machten es fast konsistent. Ohne es durch ein Tuch zu seihen, war es nicht wohl trinkbar. Und dann mußte man immer noch die Nase zuhalten, und dann schlug man sich doch noch, 
um nur die Jauche zu bekommen. An Filtrieren war für die Menge nicht zu denken. Guten ehrlichen Landmenschen kommt dies ohne Zweifel schrecklich vor. Aber wer Feldzüge und Seefahrten mitgemacht hat, findet darin nichts Ungewöhnliches » (167).

Und sein Kollege Toвias Smollet (1721-1791), den ein ähnliches Schicksal ereilte, der aber dank seiner medizinischen Vorbildung dann als Schiffschirurg etwas günstiger gestellt war, berichtete $1741 \mathrm{ganz}$ ähnliches (168): «Von den Folgen des Sieges einer Seeschlacht war uns keiner erfreulicher als der Überfluß an frischem Wasser, den wir dadurch erhielten. Fünf Wochen lang hatten wir uns mit einem Quart (etwa 1 Liter) für den Mann ganz elendiglich behelfen müssen und das unter demselben Erdstrich, wo die Sonne senkrecht über uns stand und der Körper stark ausdünstete.»

Ein entsprechendes Zitat aus dem Werk des französischen Brasilienreisenden JEAN DE LERY um 1580, also über hundertfünfzig Jahre vor SEumE und Smollet, als die Verhältnisse an Bord noch schlechter gewesen sein dürften, macht ebenfalls deutlich, daß man das ekelhaft stinkende Wasser nur unter Zuhalten der Nase überhaupt herunterschlucken konnte (99, Cap. 4, p. 33, siehe auch 154, p. 27, 54, p. 28, 87, Band 1, p. 169, 39, 126): «Outreplus (neben Würmern im Schiffszwieback) nos eaux douces estoyent si corrompues, et semblablement si pleins de vers que seulement en les tirant de vaisseaux ou on les tient sur mer, il n'y avoit si bon cœur qui n'en crachast. Mais, qui estoit bien encor le pis, quand on en beuvoit, il falloit tenir la tasse d'une main et à cause de la puanteur, bouchez le nez de l'autre (99).

Besondere Vorsicht war beim Öffnen der Fässer in geschlossenen Räumen nötig, da die entweichenden Gase gelegentlich zu asphyktischen Erscheinungen der damit beschäftigten Matrosen führten. Das Aufschlagen der Wasserfässer an Oberdeck war daher die Regel (54, p. 29). Dort wurden sie dann bei Trinkwassermangel streng bewacht (54, p. 35), da heimliches Anbohren nicht selten vorkam $(78$, p. 82).

Es ist daher kein Wunder, daß schon früh alle möglichen Versuche unternommen wurden, das notwendige Trinkwasser entweder durch andere, besser haltbare Flüssigkeiten zu ersetzen oder doch wenigstens durch Behandlung der Wasserfässer oder des Trinkwassers selbst ein einigermaßen zumutbares und nicht gesundheitsschädliches Getränk zu erhalten. Was den ersten Punkt betrifft, so ist er mit eine der Ursachen für den übermäßigen Alkoholkonsum in vergangenen Jahrhunderten an Bord (164, p. 91), Es besteht kein Zweifel, daß die erheblichen Mengen alkoholischer Getränke. 
die an Bord vorhanden waren, zum Großteil als echter Wasserersatz betrachtet wurden. Als Beispiel darf die Wasserversorgung der spanischen Armada gelten, die 1588 nur 57000 Liter Wasser, dagegen 82000 Liter Wein mit sich führte $(149$, p. 234, 161, p. 2524). Das Wasser war unglücklicherweise schon drei Monate vor der Ausreise der Flotte in defekten Fässern an Bord genommen worden und blieb zur Zeit der Schlacht im Kanal weiterhin faul, da durch die Faßspalten stets neue Keime in die Behältnisse eindringen konnten $(3$, p. 31,114$)$.

Auf holländischen Ostindienfahrern wurden pro Tag durchschnittlich eine Kanne Wasser für eine Back mit drei bis acht Mann, ebensoviel Bier und zusätzlich ein halbes bis sechs «mutjes» spanischen und französischen Weins (d.h. 0,08 bis 0,9 Liter) pro Mann ausgegeben! Pro Mann kamen auf hoher See dagegen gelegentlich nur $45 \mathrm{ml}$ Wasser pro Tag (83). Es war auch auf französischen Schiffen üblich, daß schon zum Frühstück bis zu $60 \mathrm{ml}$ Weinbrand sowie mittags und abends ein halber Liter Wein und $25 \mathrm{ml}$ Branntwein ausgeschenkt wurden (154). 1874 wurde noch pro Mann und Tag bei der französischen Marine 1 Liter Wein und $100 \mathrm{ml}$ Branntwein ausgegeben. Eine ganze Anzahl von Schiffsärzten war im übrigen der Ansicht, daß gerade Branntwein für die Verdauung der in ihrer Bewegung so außerordentlich eingeschränkten Seeleute von Nutzen sei $(34$, p. 8$)$, ja speziell gegen die Melancholie, aber auch gegen Wasser- und Gelbsucht eine Art Therapeutikum darstellte $(34$, p. 75). In einer kürzlich in einer philologischen Heidelberger Dissertation der Öffentlichkeit wieder zugänglich gemachten Anweisung über die Ernährung an Bord von Kriegsschiffen für das «Occidentalische Meer», d.h. den Atlantik, aus dem Kriegsbuch des Herzogs Philipp von Cleve, der von 1485 bis 1502 als Admiral eine Flotte befehligte, waren für eine tausendköpfige Flottenbesatzung mehr Weinund Bier- als Wasserfässer vorgesehen (144).

Jedoch soll hier auf diese Art des Flüssigkeitsersatzes nicht näher eingegangen werden. Erinnert sei nur daran, daß die südländischen Schiffe mehr Wein, die nordländischen mehr Bier mit sich führten und dieses entweder in Form von Dünnbier, das zum Teil auch mittels sogenannter Getreidesteine (Zeilolithen) mit Wasser an Bord ad hoc hergestellt wurde (180, 186, p. 61) oder auch in Form des zu selteneren Gelegenheiten verabfolgten bitteren Bieres, das heute noch in England unter dem Namen Porter läuft, ausgegeben wurde. Weiter waren bei allen Marinen, allerdings mehr als Roborans denn als Getränk, die gebrannten Wässer - seit 1700 bei der Royal Navy besonders Rum (149, p. 131, Ann., p. 240), der um 1651 in 
England bekannt geworden sein muß (89, p. 614) - beliebt. Ihr Abusus hat jedoch 1740 den englischen Admiral Edward Vernon (1684-1757), der wegen eines unansehnlichen Rockes, den er ständig an Bord trug, den Spitznamen «Groggram » erhalten hatte, veranlaßt, den seinerzeit auf englischen Schiffen beliebtem Rum mit erheblichen Mengen Wasser zu verdünnen (106, p. 89), dem später ein wenig Zucker zugesetzt wurde, um dieses neue Getränk, das bald den Namen «Grog» erhielt, der Besatzung schmackhafter zu machen $(23$, p. 235,106 , p. 88,149 , p. 240, 161, p. 2523). Er hoffte damit: "the swinish vice of drunkeness too visible increasing in H. M. Service" erfolgreich bekämpfen zu können (106, p. 88).

Die Ansicht des großen englischen Flottenarztes Sir Gilbert Blane (1749-1834) zu Ende des 18. Jahrhunderts, der im übrigen Wein und Tannenspitzenbier als beste Antiskorbutika pries (23, p. 295, 301, 307, 106, p. 90): «Matrosen haben einen unwiderstehlichen Hang zu berauschenden Getränken, welcher sich aus den großen Mühseligkeiten ihrer Lebensart und den vielen Abwechslungen und Unordnungen des Seelebens leicht erklären läßt» (23, p. 232), und die Auffassung des Bremerhavener Auswandererarztes Отто Heinrich With - Mitte des 19.Jahrhunderts: «Trunkenheit ist der Aussatz der Matrosen. Sie ist der schmutzige Sumpf, in welchem sie ihre Gesundheit ersäufen und ihre intellektuellen und körperlichen Kräfte vernichten» (186, p. 7), dürften heute nur noch cum grano salis Geltung haben.

Mit der Einführung alkoholfreier Getränke wie Tee, Kakao und Kaffee zwischen 1815 und 1830 (149, p. 130, Ann., p. 240, 106, p. 89, 57, p. 5, 58,186, p. 54,28, p. $32,51,2^{\circ}$, p. 716 und 720$)$ standen dann endlich angenehm schmeckende und bekömmliche Flüssigkeiten zur Verfügung, die das ekelhafte Trinkwasser schnell ablösten.

Versuche, die Qualität des Wassers zu verbessern, gingen einmal von der Behandlung der Holzfässer aus, die man schwefelte (13, p. 19, 32, p. 29), eine auch heute noch beim Weinbau übliche Methode, aber auch mit Öl, Pech, Harz oder Manganoxyd behandelte (186, p. 50), ohne damit auf die Dauer größere Erfolge zu erzielen, weil infiziertes Trinkwasser schnell wieder Gärungs- und Fäulnisprozesse durchmachte und Algen und Bakterien in frischem, noch eiweißhaltigem Holz einen günstigen Nährboden fanden. Später versuchte man nach einem Vorschlag von Tномаs Trotтer (1760-1832), einem anderen verdienstvollen englischen Flottenarzt, die Innenfläche der Fässer zu verkohlen, um auf diese Weise keinen Kontakt sogenannter «animalischer Stoffe» mit dem Wasser mehr aufkommen zu lassen 
(32, p. 29, 51, p. 465, 149, p. 104, Ann., p. 104, 177, Band 1, p. 52,92, p. 243, 28, p. 29, 60, 173). Die Verkohlung der Trinkwasserfässer wurde dann zu Anfang des 19. Jahrhunderts schnell bekannt, nachdem 1803 CuAdue Louis Berthollet (1748-1822) die starke Adsorptionskraft der Kohle klar erkannt hatte $(21,70)$. Dieser hatte Trinkwasser vier Monate lang in je einem verkohlten und unbehandelten $\mathrm{Fa}$ aufbewahrt. Im ersten war beim Öffnen, «l'eau... potable et sans mauvais goût», im anderen «tellement gâtée qu'on ne pouvoit en soutenir l'odeur». Die Methode bewährte sich besonders während der Weltumseglung des in russischen Diensten stehenden Admirals Adam Johann von Krusenstern (1770-1846), der in den Jahren 1803 bis 1806 insbesondere die Kurilen und Alëuten erforschte $(48,54$, p. 31, 60, 70, 91, p. 243). Das Trinkwasser soll sich auf dieser Reise nach Japan in solchen Fässern drei Jahre frisch gehalten haben $(48$, p. 232): «Daß das Wasser in den verkohlten Fässern sich unverdorben hielt, verdient noch einmal angemerkt zu werden, weil es ein Punkt von größter Wichtigkeit war, denn ohne diese Vorsicht verdirbt es in einigen Wochen und stinkt abscheulich; das unsrige hingegen war, einige wenige Fässer ausgenommen, bey denen ein Versehen angenommen sein muß, immer recht gut trinkbar.» Das Wasser bekam jedoch in verkohlten Fässern einen unangenehmen rauchigen Geschmack $(54$, p. 31$)$.

Auch das Verkupfern der Innenfläche der Wasserfässer wurde geübt, um die Trinkbarkeit des Trinkwassers zu erhöhen (22,60), bis es schließlich 1815 zur Einführung von eisernen Wassertanks kam. Dadurch wurden die unhandlichen Holzfässer, die zudem oft noch den Faulungsprozeß des Wassers überhaupt in Gang brachten oder unterhielten, abgelöst (51, p. 464 f., 54, p. 12 und 49, 16, p. 28, 3, p. 190, 58, 149, p. 104, Ann., p. 232, 154, p. 26, 188, p. 84, 20, 122,57, p. 5,186 , p. 52, 129, 153). Der Begriff «Tank» für Wasserbehälter stammt im übrigen aus dem Hindustanischen und wurde 1658 zum erstenmal in diesem Sinne im Deutschen gebraucht $(89$, p. 769, 153). Schon Vasco da Gama hatte 1498 in Melinde viereckige, mit Kokosschnüren zusammengehaltene und mit Harz abgedichtete Wasserbehälter kennengelernt, die die eingeborenen Seeleute als «tenki» bezeichneten (154, p. 30, 174).

Diese Wassertanks, die nun fest in das Schiff eingebaut werden konnten und die nach ihrer Entleerung Seewasser als Ballast aufnahmen, hatten allerdings einen Nachteil, sie rosteten schnell (92, p. 243). Während dieser Rost, der dem Wasser eine unerfreuliche rötlich-braune Farbe und einen schlechten Geschmack verlieh, von einzelnen Ärzten als ein willkommenes 
Therapeutikum gegen die Tropenanämie angesehen wurde (51, p. 467, 127, p. 226, 186, p. 50), betrachteten andere Schiffsärzte den Rost als schädlichen Zusatz, der zu gastrischen Beschwerden führen könne (51, p. 467, 51a, Bd. 1, p. 266, 54, p. 50).Autoren, die der ersten Gruppe angehörten, empfahlen sogar, die Rostrückstände - in zwei Monaten konnten sich bis zu sechs Pfund Rost ansetzen-in das Brot an Bord zu verbacken (51, p.467, 54, p. 50), und die Rostbildung wurde auch als Mittel zur Niederschlagung «organischer Materie» sehr begrüßt (185).

Versuche, die Eisentanks durch Innenanstriche mit Teer vor Rostbefall zu schützen, scheiterten an dem schlechten Geschmack, den das Trinkwasser dann annahm, oder ließen gar, wenn man, wie bei der französischen Marine, mit Vorliebe bleihaltige Farben verwandte, neue Gefahren aufkommen, auf die wir noch zu sprechen kommen werden (siehe S. 77). Ebensowenig erfüllten Email-, Mastix-, Harz- und Wasserglasbelege oder Kalkanstriche, die in der Mitte des vorigen Jahrhunderts gegen das Rosten empfohlen worden waren, die in sie gesetzen Erwartungen (51, p, 469, 129, 147, p. 565,186 , p. 54 f.). Von allen Belegen hat sich dann der ab 1863 eingeführte Zement am besten bewährt. Er ist erst in jüngster Zeit durch antibakterielle Anstriche ersetzt worden. Heute werden zumeist auszementierte Tanks oder solche aus Aluminium, Stahl oder Kunststoff, zum Teil mit antibakteriellen Anstrichen oder Verkleidungen versehen, verwendet $(169,170)$. Etwa fünfzig Jahre nach ihrer Einführung wurden die Eisentanks jedoch als einer der großen Fortschritte der Schiffshygiene gepriesen: "The general use of iron tanks on shipboard, for preserving and purifying water, is one of the great improvements of modern times" (185).

Dem Wasser selbst wurden die verschiedensten Mittel zugesetzt in der Hoffnung, damit das Salz ausfällen zu können. Schon im 17., erst recht aber im 18. Jahrhundert beschäftigten sich auch die gelehrten Gesellschaften sehr intensiv mit dem Problem der Entsalzung des Meerwassers, so 1683 die Royal Society (87, Band 2, p. 160) und 1754 die Académie de la Marine (97, p. 56). Besonderer Beliebtheit erfreute sich der ungelöschte Kalk, dessen Verwendung bei der französischen Marine 1786 obligatorisch wurde (54, p. 31), aber auch der weniger gefährliche gelöschte Kalk (23, p. 237, 102, $2^{\circ}$, p. 36), Holzasche (31), Alaun (51, p. 476), den schon die Chinesen seit ältester Zeit zur Verbesserung des Jantsekiang-Wassers benutzt haben sollen, Tierkohle $(51$, p. 476,54 , p. $34,88,32$, p. 29, 78, p. 65), deren absorbierende Kraft um 1780 entdeckt worden war, und Weinstein als «Cremor tartari» sowie Schwefelsäure, Gerbsäure, Salzsäure $\left(102,2^{\circ}\right.$, p. 36, 113, 81, 149, 
p. 107 Ann., p. 233, 23, p. 239, 59, 13, p. 19, 92, p. 241 f., 78, p. 65, 72, p. 2, 97, p. 56 f.). Im Zuge der durch James Lind (1716-1794) um 1750 eingeführten Skorbutprophylaxe wurde Limonen- oder Zitronensaft zur Konservierung des Wassers herangezogen $(59,92$, p. 241, 172). Schon vor Einführung der Eisentanks wurden sogar einige Pfund alten rostigen Eisens als Trinkwasserzusatz, Konservierungsmittel und Desodorans empfohlen (54, p. 31). Ein anderes Verfahren war das Hineinhängen eines Topfes mit grauer Quecksilbersalbe ins Trinkwasser oder der Zusatz von Teer (32, p. 29, 54, p. 32). Die Erfolge waren allerdings recht mäßig.

In anderen Fällen ließ man das Wasser durch entsprechende Sand-, Kohle- oder auch Schwammfilter durchlaufen oder benutzte Löschpapier, ja sogar Schiffszwieback dazu (51, p. 473 f, 51a, Bd. 1, p. 267 ff., 81, 100, $102,2^{\circ}$, p. 92, 131, p. 80 f.). Auch dünne Baumstammscheiben wurden, zuerst von dem österreichischen Forstingenieur Pfitzer 1894 (81), für diese Filtration benutzt ebenso wie Eisenschwämme aus porösem Fe und Braunsteinsäure, Asbest- und Tonfilter (42, 81, 92, p. 242). Mit Hilfe von Baumscheiben konnten immerhin in einer Stunde 40 Liter Trinkwasser gewonnen werden (81). Auch Leinentücher wurden als Filter verwandt (51, p. $475,102,2^{\circ}$, p. 92). Auf diese Weise gelang es, wenigstens die gröbsten übelerregenden Bestandteile aus dem putriden Wasser zu entfernen (131, p. $78,100,81,78$, p. 60,23, p. 240,92, p. 242,113 , Band 2, p. 25,66 ).

Am beliebtesten waren die Sandfilter. Schon Pictorius empfahl z. B. in seinem Raißbuechlin von 1557, trübes faules Wasser durch einen Sack voll Sand oder Kies eventuell bis zu viermal zu seihen und Alaun zuzusetzen $\left(137\right.$, fol. $\left.20^{\mathrm{r}}\right)$. Offensichtlich war auch auf den Indienseglern die Möglichkeit bekannt, an unbekannten Stränden durch Graben von Wasserlöchern am Strand leidlich salzfreies Wasser zu gewinnen (132). Diese Methode kannte auch schon Francis Bacon von Verulam (1560-1626) (12, Cent. 1, 1, p. 82). "Dig a pit upon the sea-shore some what above the high-water mark, and sink it as deep as the law water mark, and as the tide cometh in, it will full with water, fresh and potable", und bereits in den

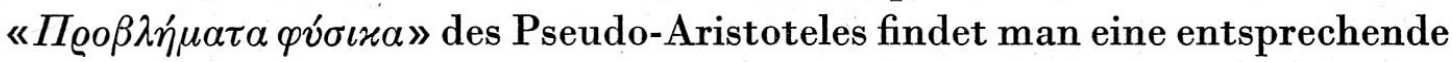
Andeutung (8, Band 2, p. 26f., Lib. 23, 21) daß Sandlöcher an der libyschen

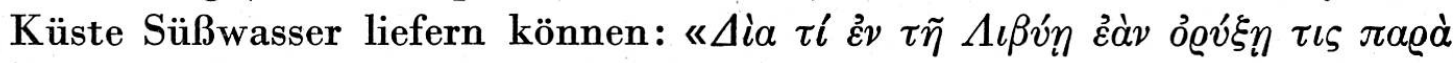

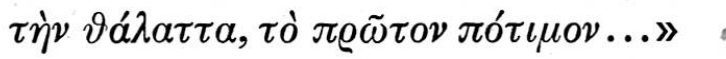

Auf holländischen Schiffen wurde eine Art Ton- oder Steinfilter, der «Lapis mexicanus», zur Filtrierung benutzt, das zwar Schmutz abhalten, aber nicht das Kochsalz entfernen konnte $(72$, p. 2). Luigi Ferdinando Graf 


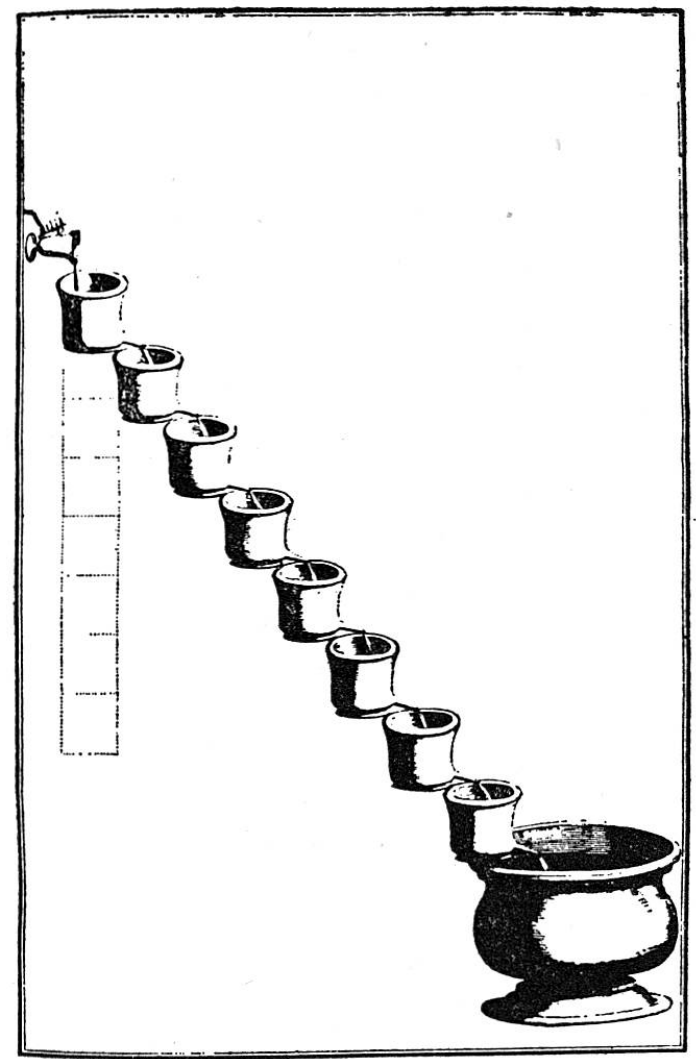

$A b b$. 1. Von Luigi Ferdinando Graf de Marsigli (1658-1730) angegebenes Filtrationsverfahren. Das Meerwasser lief durch die ganze Reihe der teils mit Erde, teils mit Sand gefüllten Tongefäße hindurch. Aus: 113, p. 32, T. 12

De Marsigli (1658-1730) versuchte 1725 eine Filtration des Seewassers mittels 9 bis 15 Steinguttöpfen, die er teils mit Erde, teils mit Sand füllte und übereinanderstellte (113) (Abb.1). Seine Entsalzungserfolge waren jedoch mehr als mäßig. Immerhin ergab sich, daß Sand sich bezüglich der Klärung des Wassers noch am besten zu bewähren schien (72, p. 2), was schon Francis BAcon feststellte: "The Sea water passing of straining through the sands, leaveth the saltness" (12, Cent. 1,1, p. 82), und Marsigli bestätigte: "Cette diminution du goût salé et du poids qui se trouve plus grande dans la filtration par le sable, qu'en celle qui se fait par terre, montre qu'il est le plus propre à purifier l'eau...» $(113$, p. 33).

Besonders der bittere Geschmack des so behandelten Wassers ließ es aber nur in seltenen Fällen genießbar erscheinen: «que bien qu'elle soit entièrement depouillée du sel, l'amertume qui lui reste la rend si dégoutante qu'il n'est pas possible de la boire» (113, p. 41). 
An dieser Stelle ist wieder LiNd zu nennen, der in der zweiten Auflage seines 1762 erschienenen Buches An Essay of the Most Effectual Means of Preserving the Health of Seamen eine recht wirksame Filtriermöglichkeit mit Hilfe eines größeren und eines kleineren Fasses angab, die beide mit Sand gefüllt wurden und durch die das Wasser hindurchlaufen konnte $(102, \mathrm{p}$. $90 \mathrm{f.,} \mathrm{3,} \mathrm{p.} \mathrm{141)} \mathrm{(Abb.2).}$

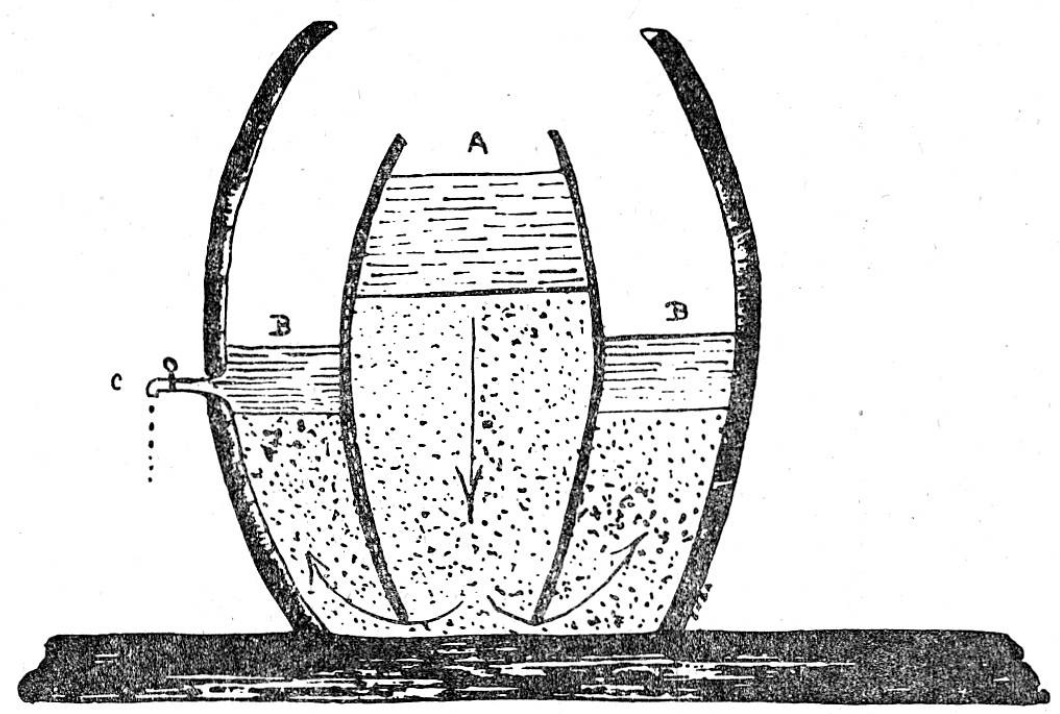

Abb. 2. Das von James Lind (1716-1794) in der zweiten, 1762 erschienenen Auflage seines Werkes An Essay on the Most Effectual Means of Preserving the Health of Seamen angegebene Verfahren der Meerwasserdestillation mittels zweier mit Sand gefüllter Fässer (102). Nach: 3, p. 142

Kein Geringerer als der berühmte Iatrochemiker JoHAnn Rudolf GLAUBER (1603-1670) hatte sogar eine Consolatio navigantium verfaßt, einen Trost der Seefahrenden, der in lateinischer, deutscher und holländischer Sprache vorliegt $(41,65,135,146,154$, p. 29, 163). In dieser erstmals 1657 herausgegebenen Broschüre wurde ein Geheimmittel angegeben, das angeblich die Seeleute vor Hunger, Durst und zahlreichen Krankheiten, «so ihnen auf langwierigen Reisen begegnen möchten», bewahren konnte. GLAUBER empfahl ein sogenanntes «konzentriertes Wasser», nichts anderes als verdünnte Salzsäure, wie wir es heute bezeichnen würden, als Zusatz zu Trinkwasser oder Bier. Dadurch sollte Fäulnis und Skorbut verhindert werden können. Weiter schien dieses Mittel eine Panazee als Diuretikum, Diaphoretikum, Analeptikum und auch ein Konservierungsmittel für Fleisch und Fisch zu sein. Bei Schiffbruch löschten einige Tropfen auf etwas Zucker angeblich vollständig den Durst. Zwar gelang es auch GLAUBER noch nicht, mit Hilfe 
dieses Wundermittels Meerwasser trinkbar zu machen. Hingegen konnte man nach seiner Auffassung in mit diesem «Aqua concentrata» versetzten Meerwasser wohlschmeckende Gerichte kochen.

Auch der in London lebende Privatgelehrte Sir Hugh Pratt (1552-1608) hatte ein solches Patentrezept zur besseren Haltbarkeit von Flüssigkeiten zur Hand: "to last (water) sweete, good, and without any intention to putrefaction, for 2, 3, or 4 yeeres together". Dahinter dürfte sich jedoch nur einfaches Kochen und Versiegeln der so behandelten Gefäße verborgen haben, also eine frühe Form der Konservierung (87, Band 1, p. 108). Kochen des Wassers war dann als Hilfsmittel auch 1767 von Kapitän Edward Thomson empfohlen worden $(150$, p. 94). Hales nahm bereits etwa fünfunddreißig Jahre früher die Versuche der Konservierung des Trinkwassers auf $(72$, p. 14), ohne grundsätzlich neue Erkenntnisse beigetragen zu haben. Ein anderes Geheimrezept des italienischen Alchemisten Leonardo FioraVANTI (gest. 1588) der im übrigen behauptete, die Meerwasserdestillation erfunden zu haben $(49$, p. 155), bestand wohl aus nichts anderem als dem Zusatz von etwas Seewasser zum Trinkwasser bzw. zum Destillat und sollte diesem überdurchschnittliche Haltbarkeit verleihen $(50,72$, p. 15).

Glauber war es übrigens auch, der eine weitverbreitete Methode der Wasserverbesserung wieder ins Gedächtnis seiner Leser zurückrief, nämlich das Eintauchen von glühendem Stahl oder glühend gemachten Kanonenkugeln, um ungenießbares Trinkwasser trinkbar zu machen $(32$, p. 29, 54 , p. 33, 110, 121, 133, 151, 153, 154). Diese Methode war ins- besondere beliebt, um dem in geteerten Segeln aufgefangenen Regenwasser den unangenehmen Geschmack zu nehmen $(19,121)$. Der schale Geschmack des Regenwassers und des destillierten Trinkwassers selbst wurde im 19. Jahrhundert dann durchaus auf das Fehlen bestimmter Mineralien zurückgeführt, und es wurden Zusatzrezepte zur Verbesserung dieser Trinkwasserquellen angegeben, in denen Kalk, Wein, Rum, Branntwein, Zitronensaft, aber auch detaillierte Mineralsalzzusätze empfohlen wurden. Hier ein solches «Rezept»:

Salzsaures Natron 4,8

Schwefelsaures Natron 3,4

Doppeltkohlensaures Natron 48,0

Kohlensaures Natron 14,0

Kohlensaure Magnesia 6,0

Davon 1 Pfund auf 480 Pfund Trinkwasser (186, p. 44) 
Eine weitere Verbesserungsmethode bestand in der Aeration durch Schlagen des Wassers mit Besen und durch Emporspritzen mit Hilfe einer kleinen Pumpe und Versprühen über entsprechenden durchlöcherten Scheiben, wodurch der schwefelartige faule Geruch vertrieben werden sollte (51, p. 472 f., 186, p. 44, 54, p. 33, 78, p. 62 f., 23, p. 241, 84, 56, 93, 95, 173, 73, 72 , p. 29). Schon Plinius erwähnte, daß Wasser durch Berührung mit atmosphärischer Luft besser und gesünder würde $(81,140)$. In Konstantinopel wurden aus diesem Grunde hohe, oben offene, gemauerte Türme als Wasserzisternen verwandt, in die die Luft leicht hineindringen konnte (81). In jüngster Zeit ist es endlich geglückt, einen nach dem Prinzip des Kationenaustausches arbeitenden handlichen Apparat für Seenotfälle zu konstruieren $(10,118)$.

Diese Methoden der Trinkwasserkonservierung sind aber von der $D e$ stillation des Seewassers überrundet worden, die allein in der Lage war, frisches Trinkwasser, das auch in den meisten Fällen gesundheitlich einwandfrei war, zu liefern. Wir erwähnten schon, daß die Destillation bereits dem heiligen BAsILIUs bekannt war (15). Sie dürfte in byzantinischer Zeit nichts Ungewöhnliches dargestellt haben. Auch im Mittelalter war diese Methode durchaus bekannt. So hatte unter anderem Gilbertus Anguicus (um 1245), der 1227 eine Reise ins Heilige Land unternommen hatte, zur «sedatio sitis» an Bord eine Destillationsmethode mittels Alembik und Sandfiltern empfohlen (62, p. 6, 120, 87, Band 1, p. 18, 136, p. 6) : «Rectificatur autem aqua per eventationem et excolationem et agitationem et nostrum aut per excoctionem et post promittatur residere, et fex abiiciatur aut coletur per arenam limpidam recentem, aut si fieri potest sublimetur per duplicia vasa scilicet per alembicum ad nobiles sic enix fit aqua salsa dulcis» (62).

Auch Andreas Laguna von Segoria (1499-1560), der Leibarzt Kaiser Karls V. (1500-1558), dürfte die reine Destillation von Meerwasser ebenso geübt haben (94) wie Johannes Angligus 1516 (4) und Sebastian de la Pollère 1560, der bei einer Belagerung durch die Türken in Sizilien täglich angeblich fünfunddreißig große Fässer mit Süßwasser auf diese Weise aus Seewasser gewinnen konnte (81, siehe 51 , p. 480, 28, p. 21, 161, p. 2525). An dieser Stelle wäre weiter wiederum der geniale, menschlich dagegen so umstrittene Francis Bacon zu erwähnen, der die Destillation zur Entsalzung des Meerwassers ebenfalls kannte (12, Cent. 9, 881, p. 187, siehe 103, p. 325): "Salt water boiled, or boiled and cooled again, is more potable."

Und Fioravanti betonte bereits, daß destilliertes Wasser sogar für 
Kranke besonders erquicklich sei $(50)$ : «è questo civè l'acqua del mare distillata e miracolosa da bere per gli enfermi.»

Bereits 1593 führte Sir Richard Hawkyns (um 1562-1622) ein Destilliergerät mit sich, das auf der «Daintie» gute Dienste leistete, als das Frischwasser knapp wurde und vor allem die Kranken mit einwandfreiem Trinkwasser versorgt werden mußten (77, 87, Band 1, p. 103, 71, Band 6, p. 410, 165, 149, p. 106, Ann., 233): "Although our fresh water had fayled us manydayes (before we saw the shore) by reason of our long Navigation, without touching any land, and the excessive drinking of the sicke and diseased (which could not be excused) yet with an invention I had in my Shippe, I easely drew out of the water of the Sea sufficient quantitie of fresh water to sustaine my poeple, with little expence of fewell; for with foure billets (of wood) I stilled a hogshead of water, and therewith dressed the meate for the sicke and whole. The water so distilled we found to be wholesome and nourishing."

Auf der Expedition von Pedro Fernandez de Quiros (1565-1615), in die Südsee, die 1606 zur Entdeckung der Neuen Hebriden und zur Namensgebung dieser Inseln als angeblichen Erdteil «Austrialia» (aus «terra australis» und "Austria» entstanden) führte, wurde ebenfalls ein kupfernes Destilliergerät mitgeführt, das zufriedenstellend arbeitete, aber wohl nur kleine Mengen Frischwasser liefern konnte $(101,158,176)$ : "6th febr. (1606): On this day the Hearth was arranged and the apparatus for retaining fresh water from salt. -7 th: The fire was lighted over the machine and it began to give fresh water with much ease. This day they got three Peruvian jarsfull. The object was to try the machine, and the water was found to be clear, soft, and good for drinking" (101).

1624 wurde der Direktion der Holländisch-Ostindischen Kompanie, den «Heeren Zeventien», von dem Amsterdamer Doktor Aegidius Snoek der Vorschlag unterbreitet, durch Destillation aus Seewasser Süßwasser zu gewinnen. Seine Methode wurde gelegentlich an Bord verwandt (142, 164, p. 91). 1670 empfahl der französische Ingenieur Hauton zu der von ihm angegebenen Meerwasserdestillation an Bord ein kombiniertes Verfahren, und zwar zuerst die Präzipitation mit Oleum tartari, dann die Destillation und schließlich die Filtration durch eine bestimmte alkaliangereicherte Erde $(28$, p. 23,54, p. $23,76,161$, p. 2526), dessen System noch im 19. Jahrhundert für ähnliche Konstruktionen herangezogen wurde und das mit Seewasser als Kühlwasser arbeitete. Dadurch sparte er den Raum für die Kühlkessel an Bord ein! Das destillierte Wasser ließ er in Bleiröhren außen- 
bords führen, um es durch Kontakt mit dem Meerwasser zu kühlen (76, 72 , p. $6,81,25,28$, p. 23,51 , p. 480 ff., 161 , p. 2526).

Um 1675 herum führte William Walcot König Karl II. von England (1630-1685) ein Destilliergerät mit langem, rohrartigen Rezipienten vor, in das er zum Abfangen der unangenehm schmeckenden organischen Begleitstoffe ein Geheimmittel einbrachte (51, p. 480, 72, p. XI, 182, 183). Um seiner Methode den Anstrich der Novität zu geben, verwandte er wohl bewußt nicht die auch damals längst bekannte Fachbezeichnung «Destillation», die schon seit dem 15. Jahrhundert gebraucht wurde (89, p. 128). Er erhielt für seine «Erfindung» vom König und den Generalstaaten ein Patent (72, p. XIII). WALCOT fand einen ihn wegen dieses Patents erbittert bekämpfenden Gegner in Fitzgerald, einem Verwandten des berühmten Chemikers Robert Boyle (1627-1691), der ein ähnlich arbeitendes Gerät 1683 erfunden haben wollte (72,p.XII, 182). Auch FitzGerald benutzte eine Absorptionsmasse zur Verhütung des schlechten Destillationsgeschmacks. Sein Apparat wurde schnell berühmt, Gedichte und Medaillen zeugten von dem allgemeinen Interesse in England, aber Versuche mit seiner «Maschine» auf den Kanalinseln Jersey und Guernsey brachten keinen zufriedenstellenden Erfolg, das Wasser schmeckte schlecht und blieb ungenießbar (72, p. XVIII). Ähnliche Geräte müssen dagegen schon um 1683 auch bei Seegang, wenn sie z.B. auf der Back vor dem Fockmast installiert waren, erfolgreich und zufriedenstellend gearbeitet haben, wie Hales berichtete, der sich ausführlich mit dieser Methode und ihrer Adaptation für die Seefahrt beschäftigt hatte (72, p. XXII). 1684 demonstrierte NeHEMIAH GREW (1641-1712) den Naval Consultants der Royal Navy einen Destillationsapparat, der einstimmige Anerkennung fand (87, Band 2, p. 160, 111): "...several eminent physicians of the College and others, have so well approved of this prepared water (d.h. Aqua dest.), that they have decided to use thereof on Diet-drinks, Coffee, Chocolate, and Thea".

Für die Zeit zwischen 1691 und 1696, als derartige Apparate schon öfter an Bord holländischer Handelsschiffe der Niederländischen Ostindischen Kompanie zu finden waren, ist eine interessante Statistik erhalten. Auf Schiffen ohne Destilliergerät betrug die Mortalität nämlich durchschnittlich 13,5\%, auf solchen, mit der Möglichkeit zu destillieren, aber im gleichen Zeitraum nur etwa 9,5\% (164, p. 91).

Ursprünglich war man jedoch der Auffassung, daß diese Meerwasserdestillation nur mit Hilfe chemischer Zusätze möglich wäre. Knochenasche, Seifensiederlauge, pulverisierte Kreide, Vitriolöl (also verdünnte Schwefel- 
säure), Gerbsäure, Kohlepulver, ja sogar Lapis infernalis, der Höllenstein, wurden zum Ausfällen von Salzen zugesetzt (28,p.24,51,p.479, 149, p. 107,85, p. 112). Kaliumpermanganat diente zur Klärung, wurde aber auch als nicht ungefährlich abgelehnt $(54$, p. 32). Ein 45 tägiger Versuch an Sträflingen, die mit Kaliumpermanganat geklärtes Trinkwasser erhielten, zeigte die Haltlosigkeit der Bedenken (51, p. 465).

1739 hatte sich auch HaLes mit dieser Destillation beschäftigt: "... to find a never-failing spring of fresh waters in the midst of the ocean" (72,p. II). Er ließ das Wasser vor der Destillation faulen und klärte es dann mit Fischleim, um den ölhaltigen Geschmack zu vermeiden. Dabei wurde nur ein Drittel des so behandelten Wassers zur Destillation herangezogen: "... the best Method to procure wholsome Water from the Sea, is first to let it putrify well, and then become sweet before it be distilled..." (72, p. 50).

Hales machte weiter Versuche mit verschiedenen Substanzen, wie Alaun, gepulverter Austernschale, Eiereiweiß, Silber, das er in Scheidewasser löste, Oleum tartari, Knochenasche und Salzsäure, um den unangenehmen Geschmack des destillierten Wassers zum Verschwinden zu bringen (72, p. $14 \mathrm{ff}$.): "Thus we see that Oil, and Salt of Tartar, Calx of Bones, OysterShells, Chalks and Brock-Dust have a good effect in curing the noxious quality of distilled Sea-Water: But this not without a second Distillation..." (72, p. 26).

HALES empfahl schließlich auch, die Destillation von Meerwasser nicht, wie sonst üblich, in Kupferkesseln vorzunehmen, da das Salz leicht zum Ansatz von oft brecherregender Patina führen könne, sondern lieber Eisenoder Zinnkessel zu benützen $(72$, p. 8).

1753 gab der Durhamer Apotheker Josua Applebey ein anderes Verfahren an, das er seit 1734 erprobt hatte $(85$, p.111, 97, p.58, 54, p.24, 149, p.233, 106 , p. 91,28 , p. $24,102,2^{\circ}$, p. 85,103 , p. 327, 116, 184). Er setzte dem Meerwasser Knochenasche oder Höllenstein und Kalilauge zu. Dadurch soll das Meerwasser den widerlichen Geschmack verloren haben. Sein Verfahren wandte wenige Jahre später Kapitän William Chapman an Bord an, als er auf einer Reise nach Nordrußland in einem Sturm den Großteil seiner Trinkwasservorräte verlor, eine Behelfsdestilliereinrichtung konstruierte und dem Destillat Seife als Lauge und zur Geschmackverbesserung Holzasche zusetzte (31): "I am firmly persuaded, that wood-ashes mixed with seawater will yield when distilled, as good fresh water as can be wished for."

Er glaubte im übrigen, daß schon Sir Richard Hawkyns einen solchen 
Holzaschenzusatz benutzt hätte, da «four billets» (Scheite) Holz zur Destillation von einem «hogshead» Wasser (etwa 240 Liter) niemals genügen könnten, und deutete diesen Passus (siehe S. 69) als Hawkyns Beschreibung der Verwendung des Holzes zur Gewinnung von Asche (31).

Den öligen Geschmack des destillierten Wassers glaubte ein anderer Experte um 1806 mit Hilfe des Zusatzes von Eisensulfat und nachherigem Niederschlag durch Kreide vermeiden zu können, der dazu noch riet, etwas Eiereiweiß dem nunmehr trinkbar gemachten Süßwasser zuzusetzen (173).

1754 setzte sich die Académie de la Marine mit diesem Problem auseinander. Der Berichterstatter, Etienne Chardon de Courcelles (1705 bis 1775), erster Hafenarzt in Brest, stellte in einem bis heute nur im Manuskript und durch eine kürzlich erschienene Dissertation in Rennes unter der Leitung von Professor Huard bekanntgewordenen Referat fest, daß es zwar mit Hilfe des Destillationsverfahrens gelänge, salzfreies Wasser zu gewinnen, daß dieses aber einen "gout douceâtre et qui prend à la gorge» annehmen würde, den er mit Recht auf organische Substanzen (bitume de la mer) zurückführte und die bei der einfachen Destillation damals nicht entfernt werden konnten $(97$, p. 58). Diese «Distillation à l'alembic», wie die Franzosen es nannten, wurde ohne chemische Zusätze erst 1761 von LiND eingeführt (28, p. 25, 63, 82, 106, p. 91,30 , p. 38,54 , p. 25, 150, p. 94, 103, p. 323, 102, 2。, p. 86), nachdem schon 1717 von Jean GaUtier (1679-1743) in Lorient gezeigt werden konnte, daß man mit Hilfe einer geeigneten Apparatur, der "usine distillatoire», auch an Bord sehr wohl relativ große Mengen von geeignetem Trinkwasser ohne jeden chemischen Zusatz herstellen konnte $(28$, p. 24, 51, p. 480, 154, p. 29, 16, p. 27). Dabei nahm er sich die Sonnenwärme zum Vorbild und konstruierte seinen Apparat in der Form einer Drehtrommel nach dem Prinzip des Tauchsieders. Er ließ erhitzte Röhren in das Meerwasser selbst eintauchen und dort rotieren, um es zum Verdampfen zu bringen. Der Dampf wurde in einem zweiten Gefäß wieder aufgefangen $(54$, p. 24, 69, p. 82, 188 , p. 20, 30, p. 38, 162, 163). Das gewonnene Trinkwasser, womit einen Monat lang die Besatzung des Kriegsschiffes «Triton» versorgt werden konnte, war, nach Augenzeugenberichten, «aussi légère que celle de la meilleure fontaine du port de Lorient» (54, p. 24). Auch Lind glaubte, die Sonnenstrahlen mit Hilfe eines Parabolspiegels für die Destillation ausnützen zu können, ohne jedoch praktische Versuche angestellt zu haben $\left(102,2^{\circ}\right.$, p. 85$)$. 
Verschiedene Erfinder kämpften schließlich um die Anerkennung ihrer Priorität $(28$, p. 26, 51, p. 481). Neben Lind wäre hier noch sein englischer Gegenspieler George James Irving (1722-1798) zu nennen, dessen Destillationsapparat durch Überleitung heißer Dämpfe aus der Kombüse gespeist und mit Meerwasser gekühlt wurde und der um 1770 einen Kessel erfand, in dessen einem Teil die Nahrungsmittel mit Meerwasser gekocht wurden, in dessen anderem Teil dagegen dieses Seewasser überdestilliert

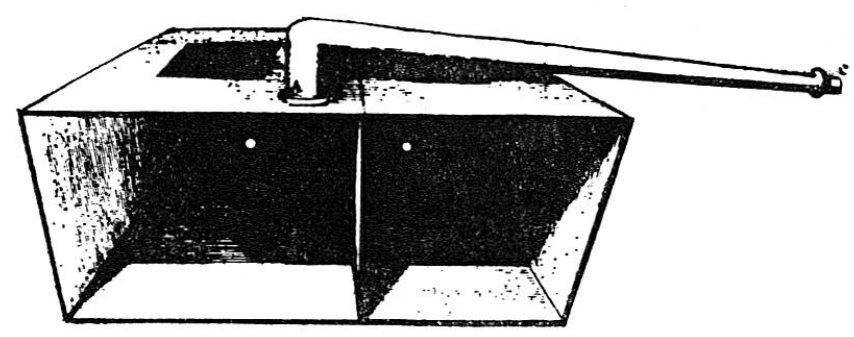

$A b b$. 3. Destillationsapparat nach George JAmes Irving (1722-1798) mit geteiltem Wasserkessel zur Destillation von Meerwasser und zum Kochen von Nahrungsmitteln und seewassergekühltem Rezipient. Nach: 85, T. VIII

werden konnte $(106$, p. $91 \mathrm{f} ., 85$, siehe auch $81,137,136$, p. 23,78 , p. 57 , 88), während LiND nur mit einem Behälter arbeitete, aber eine eigene Feuerstelle benötigte (81). Inving bekam für diese relativ geringfügige Verbesserung vom englischen Parlament 1772 einen erheblichen Geldpreis zuerkannt (150, p. 102). Lind hatte seinen Apparat offensichtlich im Jahre 1761 in Portsmouth, allerdings im Gegensatz zu GaUtier wohl nicht an Bord sondern an Land, Mitgliedern der Royal Academy vorgeführt (103, p. 324, $102,2^{\circ}$, p. 86, 150, p. 97). In der zweiten Auflage seines Werkes An Essay on the Most Effectual Means of Preserving the Health of Seamen von 1762 wurde dann seine Erfindung publiziert $\left(102,2^{\circ}\right.$, p. 85). Ein Jahr später wurde aus Wassermangel das mitgeführte LiNDsche Destilliergerät auf dem Truppentransporter «Dorsetshire» vier Stunden in Betrieb gesetzt. Man erzeugte aus 22 Quarts Seewasser (24,7 Liter) 19 Quarts destilliertes Wasser (fast 23 Liter) (150, p. 98). Linds Apparat hatte sich auch auf der Weltreise Kapitäns SAmueL WaLlis, eines direkten Vorläufers Coors, auf der «Dolphin» 1766 bis 1768 bewährt. Mit ihm konnte innerhalb fünf Stunden aus 56 Gallonen Seewasser (252 Liter) 42 Gallonen (189 Liter) trinkbares Süßwasser gewonnen werden (102, p. 105, 103, p. 66, 106, p.91). Соoк bediente sich, ebenso wie Constantin 
John Phipps, der spätere Lord Mulgrave (1744-1794) (137), dieses Gerätes auf seiner zweiten Reise. Täglich konnten damit 30 bis 40 Gallonen ( 135 bis 180 Liter) Trinkwasser gewonnen werden (54, p. 26, 154, p. 30, 51, p. 488, 52, 53). Der Generalinspektor des Gesundheitsdienstes der französischen Marine, Pierre Isaac Poissonnier (1720-1799), ein Bruder des schon erwähnten französischen Marinearztes, der 1763 ein ganz ähnliches Gerät mit Helmdeckel und Schlangenrohr entwickelt hatte, das allerdings noch mit pflanzlichen Alkalien arbeitete, war vom französischen König mit einer Dotation von 6000 Louisdor ausgezeichnet worden (16, p. 27, 136, p. 14, 106, p. 91, 117, p. 41, 134, 28, p. 25, 81). Sein Apparat wurde von Louis Antoine de Bougainville (1729-1811) auf dessen Weltreise 1766 bis 1769 mitgeführt, ohne indessen öfter benutzt worden zu sein (54, p. 25, 188 p. 20).

Ein spanischer Autor, Pedro Maria Gonzalez (1763-1838), verband Ende des 18. Jahrhunderts die Vorteile der Apparate von GaUtrer und Poissonnier, indem er das Destillationsgerät 'gleichzeitig für Back- und Kochvorgänge einrichtete $(68,81)$. Eine weitere Variation führten die französischen Marineapotheker Rocher und PeYre 1841 ein (148). Rocher erhielt für diese Apparatur 1850 einen Preis der Pariser Académie des Sciences, und die Erfinder konnten 1853 die Ausstattung der französischen Kriegs- und zum Teil der Handelsflotte mit ihrem Gerät erleben (81). Diese Apparatur war sehr klein und konnte in einer Stunde 60 bis 70 Liter destilliertes Wasser produzieren; selbst auf hoher See betrug die Ausbeute noch etwa 40 Liter, was im Notfall für eine Besatzung von 20 bis 30 Mann als Trinkwasser gerade ausreichte $(25,81,148,154$, p. 30).

Von der Mitte des 18. Jahrhunderts an waren solche Destillationsapparate vor allem auf den zu Forschungs- und Weltreisen auslaufenden Schiffen fast stets zu finden $(188$, p. $84,55,117$, p. 74 und 47$)$. Sie haben zum Teil hervorragend gearbeitet und sind im Gebrauch, wie ihre Erfinder und Befürworter immer wieder betonten, nicht viel teurer gewesen als das in fremden Häfen aufgenommene und dazu noch suspekte Frischwasser. Die Ausbeute war nun beträchtlich. Sie betrug z. B. bei Dampfschiffen bis zu 15 Tonnen in 24 Stunden und in einer Stunde etwa 650, in einer Minute um 10 Liter. Dabei war der Preis durchaus tragbar. Er belief sich bei einem Kohlenverbrauch von $1 \mathrm{~kg}$ für 3 Liter destillierten Wassers auf etwa 15 Francs für die Tonne oder 0,015 Franc pro Liter (25). Der bedeutende französische Schiffshygieniker Jean-Baptiste Fonssagrives (18231884) kam sogar auf einen Preis von nur 10 Francs pro Tonne Süßwasser, freilich legte er als Preis einer Tonne Kohle nur 20 Francs zugrunde, 
während außerhalb Frankreichs 1 Tonne bis zu 50 Francs kosten konnte $(25,51$, p. 491$)$.

Dazu konnten die Apparate allmählich kleiner gehalten werden, denn tatsächlich war die Größe der Geräte lange Zeit ein wesentliches Handikap. Weitere Schwierigkeiten bedeutete der erhebliche Mehrverbrauch an Brennstoffen, die ja an Bord ebenfalls mitgeführt werden mußten, und die Rauchentwicklung. Schon 1739 machte allerdings HALES darauf aufmerksam, daß bei Benutzung geeigneter Apparate, wie sie an Land üblich waren und bei Verwendung hochwertiger Kohle (anstatt von Holz) aus Newcastle (72, p. 9) mehr Frischwasser, als dem mitgeführten Kohlengewicht entsprach, destilliert werden konnte, also die Schiffe in Wirklichkeit sogar weniger belastet wurden $(72$, p. 5$)$. Nur hätte sich wohl damals kein verantwortungsbewußter Kapitän getraut, ohne genügende Süßwasserreserven lange, ungewisse Seereisen anzutreten.

Eine weitere Schwierigkeit bestand in der Tatsache, daß die Destilliergeräte bei Seegang nicht in Betrieb gehalten werden konnten, weil einmal die Feuerstellen gelöscht werden mußten, zum anderen oft das Meerwasser in das Destillat überlief (55). Man benutzte dagegen eine durchlöcherte Metallplatte, die bei Seegang das Meerwasser zurückhielt (85, p. 113), eine

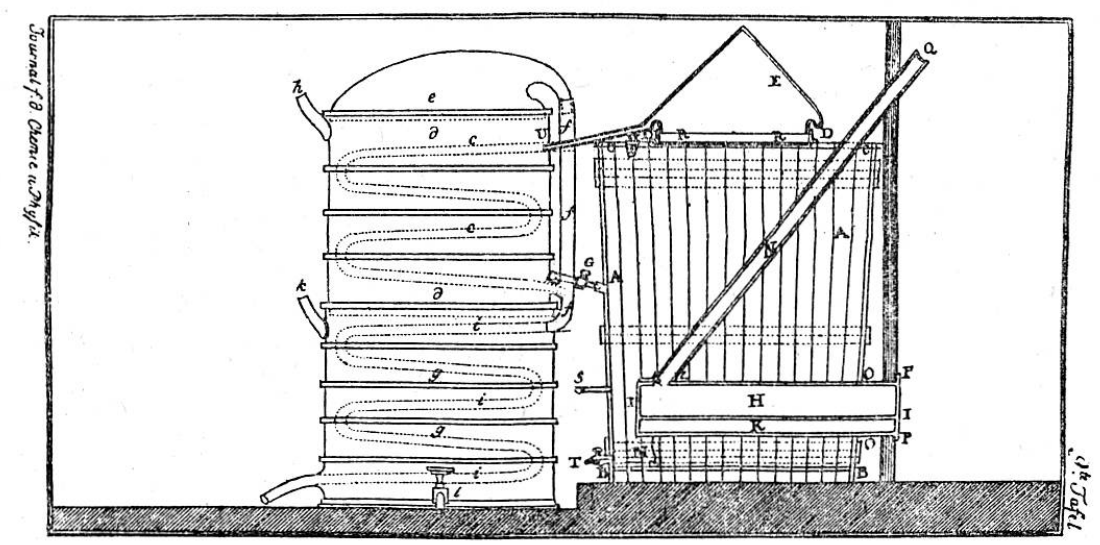

Abb. 4. Querschnitt durch ein Anfang des 19. Jahrhunderts benutztes Seewasserdestilliergerät. $A=$ hölzerne, mit Eisenreifen umschlossene Wanne, die Meerwasser aufnimmt. $E=$ Destillationshelm. $H=$ Feuerplatz, der von der Kombüse aus geheizt wird. $N=$ Schornstein. $R=$ Eiserne, mit Löchern versehene Platte, die Überschwappen des Seewassers bei Seegang verhüten soll. $d+g=$ Übereinandergestellte Fässer, die das Kühlwasser aufnehmen und durch die die Destillationsschlange ( $i$ bzw. $c)$ geleitet wird. $h=$ Einfüllöffnung für Kühlwasser. $l=$ Ausflußöffnung für Kühlwasser. Aus: 60, Fig. 9 
Konstruktion, die Louis de Freycinet (1779-1842), ein französischer Marineoffizier, perfektionierte $\left(55\right.$, siehe auch $102,2^{\circ}$, p. 87). Sein Gerät wurde auf der «Naturaliste» während einer Reise nach Australien und später auf der Weltreise der von ihm kommandierten «Uranie» 1817 bis 1820, die mit dem unglücklichen Schiffbruch des Schiffes endete, mitgeführt (55, $56,81,117$, p. 84 und 47,188 , p. 84). (Abb. 4).

Nach wie vor aber war das so gewonnene Wasser oft im Geschmack zu beanstanden. Erst die nochmalige Filtration durch Tierkohle und die Aeration änderte dies entscheidend. Die Süßwassergewinnung mittels der Elektrolyse, die T. L. Phipson bereits in Ostende 1858 bis 1862 empfahl, brachte neue Möglichkeiten (138). Große Süßwassermengen konnten aber wegen der schnellen Verschmutzung der Elektroden auf diesem Wege auch nicht gewonnen werden (81). Die in der zweiten Hälfte des 19. Jahrhunderts benutzten Destillationsapparate hatten den Vorteil, bei einfacher Handhabung destilliertes Wasser infolge erhöhten Druckes schon bei niedrigeren Temperaturen zu liefern. Die Aeration war eine weitere Verbesserung. Dabei konnten bis zu 20 Liter Luft durch 1 Liter kondensiertes Wasser getrieben werden. Der schlechte, zum Teil brecherregende Geschmack kam, wie man inzwischen erkannt hatte, einmal von Ölrückständen der Apparate, aber auch, wie schon früher vermutet, von organischen Bestandteilen des Meerwassers her (81).

Erst die Einführung der Dampfschiffe hat das Destillationsproblem an Bord völlig gelöst und die ständige Angst vor einem Brand bei Seegang verschwinden lassen, weil nun stets genügend heißer Dampf aus der Maschine zu Destillationszwecken zur Verfügung stand (136, p. 23). Damit glaubte man endgültig, den Schwierigkeiten der Trinkwasserversorgung an Bord Herr geworden zu sein, und allgemein teilte man die Auffassung: «La question de l'eau distillée est un des triomphes de l'hygiène navale» (54, p. 46). Ein besonderer Vorteil der Destillation war zweifelsohne, wie Louis H. Roddis, der amerikanische Historiker der Schiffsmedizin, mit Recht betont, die gleichzeitige Sterilisation etwa infizierten Wassers (149, p. 105, Ann., p. 233).

Das destillierte Wasser an sich war im übrigen noch im 19.Jahrhundert vielen Ärzten in gesundheitlicher Hinsicht suspekt $(52,147$, p. 657, 157). Der französische Autor Balthazar Georges le Sage (1740-1824) z.B., der sich 1817 zum Sprecher dieser Gruppe machte, war der Auffassung, daß die Existenz eines bestimmten Gases, des «Gaz alcalin oléaginé neptunien», im Meerwasser dem destillierten Wasser schädliche Eigenschaften 
verlieh $(28$, p. 27,51, p. 483,157$)$ : «...qu'on ne peut admettre l'innocuité de l'eau de mer distillée parce qu'elle contient toujours du gaz alcalin oléaginé neptunien produit par la putrefaction des êtres organisés marins» (157).

Schon lange vor ihm hatte dagegen HALES die Schädlichkeit des destillierten Wassers aufgrund des Fehlens «nährender Substanzen» behauptet (72, p. 49): "Some are of Opinion, that distilled Water cannot be wholsome, because they suspect that it is thereby deprived of its nourishing Quality."

Diese Gefährlichkeit des destillierten Wassers wurde noch anfangs des 20. Jahrhunderts auch von deutschen Autoren aufgrund von Tierversuchen angenommen, die natürlich auf die Bedingungen an Bord nicht hätten übertragen werden dürfen $(90,128)$. So schrieb einer von ihnen, der Privatdozent der Medizin in Gießen, Hans Koeppe, 1898: «Dieselbe Giftwirkung auf Zellen (wie vorher bei Protozoen behauptet) muß zutagetreten beim Trinken von destilliertem Wasser» (90).

Immerhin bedurfte es erst eines Großversuchs an einundvierzig französischen Gefangenen auf der Ile d'Henet, denen man einen Monat lang als Getränk ausschließlich destilliertes Wasser verabreichte, um festzustellen, daß dieses Wasser bei ausreichender Ernährung keineswegs gesundheitsschädlich war $(28$, p. $27,20,51$, p. 484 und 495, 13, p. 20, 81, 161, p. 2526). Diese Versuche waren von dem französischen Marschall Laurent Gouvion SAINT Cyr (1764-1830) 1817 angeregt worden, der durch Rundschreiben an die Intendanten von Brest, Toulon und Rochefort Versuche mit destilliertem Wasser an freiwillig sich dafür meldenden Galeerensträflingen empfahl (54, p. 42) und worüber der Generalinspekteur der französischen Marine PierReFrançois Keraudren (1769-1857) berichtete (88a).

Damit war der Destillation endgültig Tor und Tür geöffnet. Ein weiteres, mit der Destillation im Zusammenhang stehendes Problem war jedoch das beunruhigende Auftreten der sogenannten "Colique sèche des vaisseaux», eine Erkrankung, die endgültig erst 1859 von AMÉDÉE LEFÈvre (1798-1869) aufgeklärt werden konnte $(96,119,44,133,57$, p. 101, 1, 154, p. 30), nachdem schon 1837 Alexandre SÉgond (1799-1841) sie auf eine Bleivergiftung an Bord zurückgeführt hatte (154, p. 30, 166). Es stellte sich nämlich heraus, daß die häufigen epidemieartig auftretenden kolikartigen Durchfallsstörungen der Besatzungen französischer Kriegsschiffe nichts anderes als Folgen einer Bleivergiftung waren, die infolge des mit Essig, Zitronen- oder Obstsaft zwecks besserer Haltbarkeit und Erzielung eines angenehmeren Geschmacks angesäuerten Wassers und seines Durchlaufs durch Bleiröhren eintrat, dagegen bei der englischen Marine, wo keine bleihaltigen Geräte zur 
Trinkwasserversorgung benutzt wurden, praktisch unbekannt war. Man hatte alle möglichen Ursachen angeschuldigt. Der schon erwähnte deutsche Arzt Wiтн hielt z.B. das Krankheitsbild noch 1858 für die Folgen der Ausdünstungen des Steinkohlenteers auf Dampfern (186, p. 88.). Ausführlich ist die interessante Geschichte dieser Erkrankung in einer französischen Dissertation behandelt worden (119). Heute werden die verschiedenen Süßwassersysteme an Bord streng unterschieden, und es bestehen ausführliche Bestimmungen über das getrennte Fahren von Speisewasser für die Maschinen, von Waschwasser, von Trinkwasser sowie von Meerwasser für Feuerlöschzwecke und Abortspülungen und eventuell für Duschen. Bleirohre oder bleihaltige Farben werden an Bord nicht mehr benutzt. Toxische oder bakteriologische Verunreinigungen sollten daher praktisch nicht mehr vorkommen. Aber neuesten Arbeiten aus dem Kieler Hygienischen Institut ist zu entnehmen, daß auch heute noch die Bakterienzahl, insbesondere von Bact. coli, von $W^{\top}$ asser aus Wassertanks, selbst bei der deutschen Bundesmarine, relativ hoch sind $(45,118,125,169,170,171)$. Daher stehen zurzeit desinfizierende Anstriche, die natürlich nicht gesundheitsschädlich sein dürfen, oder eine besondere Sterilisation des Wassers durch Ultraviolettlicht, die wohl auf deutschen Schiffen erstmal von der Hapag eingeführt worden sein dürfte $(122,28$, p. 30), Einblasen von Ozon, die Trinkwassersilberung (26) oder die alte, aber unbeliebte Chlorkalkung nach wie vor im Mittelpunkt der Diskussion $(169,170,171)$.

Der Trinkwassernachschub in arktischen und antarktischen Gebieten wurde im übrigen durch die erfolgreiche Verwendung von Eisschmelzwasser erstmals durch CooK gesichert $(36,51$, p. 455, 173, 54, p. 22, 143, 131, p. 81, 78 , p. 60, 137). Damit schien endlich die alte hippokratische These von der Unverwendbarkeit des Schnee- und Eisschmelzwassers widerlegt (siehe S. 49). Cook hatte nämlich unter dem 9. Januar 1773 berichtet (36): "There (im südlichen Eismeer) we brought to, heisted out three boats, and, in about five or six hours, took up as much ice yielded fifteen tons of good fresh water ... The salt water which adhered to the ice, was so trifling as not to be tasted, and after it had lain on deck a short time entirely drained off, and the water which the ice yielded, was perfectly sweet and welltasted."

Schon 1697 hatte Samuel Reyer (1635-1714) mitgeteilt, daß Seewasser durch Gefrieren süß würde $(145,81,51$, p. 479, 28, p. 30), aber erst Cook konnte dann dieses Wissen für die Schiffahrt nutzbar machen $(36,137)$. Die Ansicht, daß das Eis der Eisberge aus Süßwasser bestünde, vertraten 
im übrigen auch schon 1757 JACOB voN WAIZ (1698-1777) (181) und 1762 LinD $\left(102,2^{\circ}\right.$, p. 91$)$ sowie der russische Naturforscher Michael Wassiljewitsch Lomonossow (1711-1765) (47, 107, 108, 109): «Wenn diese Eisberge schmelzen, wird eine Menge süßes Wasser daraus, das sich mit dem salzichten nicht vermenget, sondern oben auf demselben steht» (181, p. 43).

Schon lange vor Cook benutzten auch z.B. Amsterdamer Bierbrauer Meereis als Brauwasser (131, p. 81). Freilich hatte das Eiswasser nach Cooks eigenen Erfahrungen, die erst im 20. Jahrhundert wieder bestätigt werden konnten $(75,128)$, einen merkwürdigen Einfluß auf die Zunahme von Halserkrankungen, die zu seiner Zeit mit dem Freiwerden "fixer Luft» aus dem Wasser nach dem Schmelzen erklärt wurden $(36,51$, p. 455, 52, 53, 54, p. 23, 154). Seltsamerweise findet sich dieser Hinweis nicht in der ersten englischen Ausgabe seines Reiseberichts, sondern erst in der 1778 erschienenen französischen Fassung, die auch sonst gewisse Unterschiede zum englischen Originaltext erkennen läßt. In der französischen Übersetzung heißt es (36): »Seulement comme l'air fixe en avait chassé tous ceux qui en burent, éprouvèrent une enflure dans les glandes de la gorge; l'eau de neige ou de glace produit toujours cet effet.»

Damit war in gewisser Weise die hippokratische Warnung wieder aktualisiert worden (siehe S. 49). Cоoкs wissenschaftlicher Reisebegleiter JoHANN Reinhold Forster (1729-1798) äußerte sich dazu ebenfalls wie folgt (53): «Dieser Mangel dieses Bestandtheils (die «feste Luft») aber scheint auf die Drüsen im menschlichen Körper von nachtheiliger Würkung zu seyn.»

Noch in der Mitte des 19.Jahrhunderts war man der Auffassung, daß «luftfreies Wasser ungesund, darmerschlaffend wäre» $(81,88)$ und daß der hinzutretende Sauerstoff krankmachende organische Bestandteile des Wassers oxydieren könne $(71,51$, p. $21 \mathrm{ff}$.) .

Eine völlig andere Methode des Flüssigkeitsersatzes bei akutem Frischwassermangel war die Empfehlung, in derartigen Notsituationen im Seewasser zu baden oder die Kleidung mit Meerwasser zu benetzen, da der Körper durch die Haut in einer Art Filtrationsprozeß nur Süßwasser durchlassen würde. 1755 sollten Matrosen, die sich dieses. Verfahrens bedienten, acht Tage länger als ihre schiff brüchigen Kameraden gelebt haben und dadurch gerettet worden sein $(131$, p. 85).

Das Problem der Trinkwasserversorgung hat heute ein anderes, mehr bakteriologisches Gesicht bekommen, aber es ist auch heute noch nicht vollständig bewältigt $(125,169,170)$. Hoffentlich werden sich Katastrophen wie die auf der «Victory» 1589 und die des Schiffbruchs der «Méduse», 
einer französischen Korvette, die 1816 vor der senegalesischen Küste strandete, nicht wiederholen. Die "Victory» konnte auf den Azoren wegen der feindlichen Haltung der portugiesischen Behörden kein Trinkwasser fassen, hatte kein Destilliergerät an Bord - das damals nur ganz gelegentlich mitgeführt wurde - und kreuzte wegen widriger Winde lange auf hoher See. Sechzehn Tage sollte angeblich weder Trinkwasser noch Bier, Wein oder andere Flüssigkeiten zur Verfügung gestanden haben, und es starben wesentlich mehr Besatzungsmitglieder an Durst als an Hunger oder Krankheiten (87, Band 1, p. 118, 123). Aus Verzweiflung tranken einzelne Männer Seewasser, aber das beschleunigte nur ihren plötzlichen Tod (123, Band 5, p. 180): "Many drink salt water, and those that did died suddenly and the last word, they usually spoke, was drink, drink, drink".

Bei der Strandung der «Méduse» konnten sich 159 Passagiere und Besatzungsmitglieder dieses Schiffes auf ein Floß retten. Die Schiffbrüchigen waren jedoch ohne jedes Trinkwasser, hatten dagegen noch alkoholische Getränke mit sich genommen, die zur Löschung des brennenden Durstes benutzt wurden. Nach wenigen Tagen lieferten sich die bereits halluzinierenden und vom Alkohol berauschten Schiffbrüchigen eine erbitterte Schlacht. 63 Menschen kamen dabei ums Leben. In den dreizehn Tagen des Treibens auf dem südlichen Atlantik spielten sich entsetzliche Szenen ab. Durst ließ manchen in der wahnhaften Vorstellung, sich bereits an Land zu befinden, in das Meer stürzen. Andere begingen aus verzweifeltem Hunger Anthropophagie. Nur 15 Personen wurden schließlich gerettet, von diesen starben noch 5 an Entkräftung. Ein mitreisender Chirurg, J.B. SAvignY (1793-1843), hatte diese furchtbaren persönlichen Erlebnisse zum Anlaß einer längeren Dissertation genommen, mit der er 1818 seinen Doktortitel in Paris erwarb, und der französische klassizistische Maler ThÉodore GÉricault (1791-1824) hatte 1817 das erschütternde Erlebnis, das seine Zeitgenossen außerordentlich beschäftigt hat, in einem jetzt im Louvre hängenden Bilde festgehalten $(14,67,159)$.

Heute ist von einem Trinkwassermangel an Bord nicht mehr die Rede, jedoch beweisen, wie zu Beginn dieser Ausführungen erwähnt, Ausnahmesituationen immer wieder, daß der Klageruf des Dichters "Water, water everywhere, nor any drop to drink" (35) auch heute noch grausame Wirklichkeit werden kann. 


\section{Literaturverzeichnis}

1 Ackerknecht E. H., The Naval Surgeon, Ciba Symposia 4 (1942/43) 1407.

2 Alexander von Aphrodisias, in: Meteorum Aristotelis Commentarii, Lib. 2, Cap. 16, Venedig 1556 , fol. $21^{\mathrm{v}}$.

2 a Alr Abbas, Liber medicinalis practica et theorica, Lib. 5, Cap. 29, fol. 37a, Cap. 34, fol. $38 \mathrm{~b}$.

3 Allison R. S., Sea Diseases, London 1943.

4 Anglicus Johannes (J. Gaddesden), Rosa anglica practicae medicinae, Lib. 5, Pavia, Bd. 2, 1492, fol. $173^{\mathrm{r}}$.

5 Anson G., Voyage autour du monde, fait dans les années 1740, französische Übersetzung, herausgegeben von R. WALTER, Amsterdam 1751, p. 38.

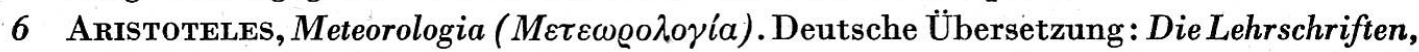
herausgegeben von P. GoHLKe, Band 4, 3, Paderborn 1955, p. 79. Englische UUbersetzung: Works, herausgegeben von W. D. Ross, Band 3, Oxford 1931, p. 359a, Lib. 2, Cap. 3, Z. 35-36, p. 354b, Lib. 2, Cap. 2, Z. 19.

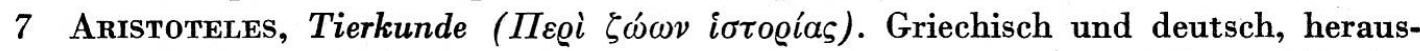
gegeben von H. Aubert und F. Wimmer, Bd. 2, Leipzig 1868, Lib. 8, Cap. 2, 21, p. 120 f.

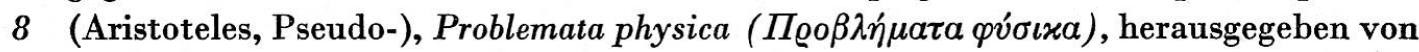
W. S. Hetr, Loeb Classical Library, London-Cambridge 1937. Lib. 23, Cap. 37, p. 36 f.

9 Athenaios, Deipnosophists, griechisch mit englischer Übersetzung von G. B. Gulick, Loeb Classical Library, Band 1, London-New York 1927, Band 2, 1928.

10 Aury J., La survie des rescapés d'un naufrage. Evolution des idées et connaissances actuelles, Rev. Méd. Nav. 12 (1957) 209.

11 Avicenna, Canon medicinae, herausgegeben von A. Alpago, Pars II, Cap. 56, Cantica, Cant. 861-870, Venedig 1555, fol. 589.

12 Bacon F., Sylva sylvarum or A Natural History, in: Works, London 1879.

13 Baldini F., La sanità dei naviganti ad uso dell'Armata Navale di S. M. Siciliana, Neapel 1789, p. $19 \mathrm{ff}$.

14 BARTET, Quelques notes biographiques sur Savigny, second chirurgien-major à bord de la Méduse, Chron. Méd. 19 (1912) 265.

14a Bartholin T., Tractatus in Peregrinatione, Kopenhagen 1674, p. 67.

15 Basilius, Homiliae IX, in: Opera omnia, herausgegeben von J. GarNIER, Hom. 4, Cap. 6, Band 1, Paris 1839, p. 54f. Ausgewähltes Schrifttum des heiligen Basilius des Großen, herausgegeben von V. GRöNE, Band 1, Kempten 1875, p. 84f.

16 Bauer W., Geschichte des Marinesanitätswesens bis 1945. Marine-Rundsch. Beiheft 4 (1958).

17 Bayley W., Description Of a Machine For Making Fresh Water From Sea-Water, Repert. Arts Manufactors 5, p. 320.

18 Behmann, Der Schiffbrüchige in physiologischer Sicht, Mitt. dtsch. Ges. Verkehrsmed. 1960, H. 7, p. 1.

19 Behr J. van de, Reise nach Java, Vorderindien, Persien und Ceylon 1641-1650, in: Reisebeschreibungen von deutschen Beamten und Kriegsleuten im Dienst der Niederländischen West- und Ostindischen Kompagnien, herausgegeben von S. P. L'HovorÉ NABER, Band 4, Den Haag 1930, p. 27. 
20 BeLli C. M., Die Entwicklung der Schiffshygiene im 19. Jahrhundert, Arch. Schiffsu. Tropenhyg. 7 (1903) 19.

21 Berthollet C.L., Mém. Inst. nat. Sci. Arts (Paris) 1803.

22 Bentham, Repert. Arts. Manufactors 16, p. 238.

23 Blane G., Observations on the Diseases Incident to Seamen, London 1785. Deutsche Übersetzung: Beobachtungen über die Krankheiten der Seeleute, Marburg 1788.

$23 a$ Boerhafye H., Elementa chimica, Band 1, Basel 1745, p. $610 \mathrm{ff}$.

24. BомваRD A., Rapport technique de l'experience de survie prolongée en mer à bord de l'Hérétique en 1952, Paris 1954.

25 Bourel-Roncière, Etude sur les appareils distillatoires de la Circé, Arch. Méd. Nav. 10 (1863) 192.

26 Braune J. F., F. Krusche, L. Kurth, H. Lippelt, H. Woratz und E. Thofern, Die Trinkwassersilberung, München 1957.

$26 a$ Browning Q., The Journal of Sir George Rooke, 1700-1702, Navy Records Soc. 9 (1897) 126.

27 Burchett J., A Complete History of the Most Remarkable Transactions at Sea from the Earliest Times to the Conclusion of the Last War With France ..., London 1720, p. 126.

28 Busche R., Beiträge zur Geschichte der Schiffshygiene, Med. Diss., Düsseldorf 1939.

29 Butler T., A Safe, Easy and Expeditious Method of Procuring Any Quantity of Fresh Water at Sea, London 1755.

30 Chaligne C., Chirurgiens de la compagnie des Indes. Histoire du service de santé de la Compagnie (1664-1793). Thèse méd., Paris 1961.

31 Chapman, W., An Account of the Distillation Water Fresh From Seawater by Wood Ashes, Philosophical Trans. 50 (1758) 635.

31a Chardon de Courcelles E., Mémoires sur le régime végétale des gens de mer, Nantes 1780

32 Chiche M. C., Hygiène et santé à bord des navires négriers au XVII siècle, Thèse méd., Paris 1957.

33 Clark J., Observations on the Diseases Which Prevail in Long Voyages to the Hot Countries, London 1773. Deutsche Übersetzung: Beobachtungen über die Krankheiten auf langen Reisen nach heißen Gegenden ..., Kopenhagen/Leipzig 1778, p. 40.

34. Cockburn W., An Account of the Nature, Causes, Symptoms and Cure of the Distempers That Are Incident in Seafaring People, London 1696. Deutsche Übersetzung: Tractat von Seekranckheiten, deren Eigenschaften, Ursachen und Cur ..., Rostock 1726, p. 8.

35 Coleridge S.T., The Ancient Mariner, Tom. II, in: Poems, herausgegeben von E. DowDEn. Edinburgh s.a., p. 43.

36 Cooк J., A Voyage Toward the South Pole and Round the World, Band 1, London 1784, p. 37. Französische Übersetzung: Voyage dans l'hémisphère austral et autour du monde, Band 1, Paris 1778, p. 114.

37 Critchley M., Shipwreck-Survivors. A Medical Study, London 1943.

38 Critchley M., Results of Exposure After Shipwreck and the Like, in: Medicine and Pathology. History of the Second World War, herausgegeben von V. Z. Cope, London 1952 , p. $295 \mathrm{ff}$.

39 Dampier W., New Voyage Around the World, London 1697. Französische Übersetzung: Nouveau voyage autour du Monde, Amsterdam 1698, p. 388. 
40 The Danger for Drinking of Sea Water and the Standard of Drinking Water, Ship Bureau, Ministery of Transportation, Japanese Government, Tokio 1960.

41 Darmstaedter E., Johann Rudolf Glaubers «Consolatio Navigantium», Münch. med.Wschr. 72 (1925) 1565.

42 Davids, Über die Verwendung von Filtrierapparaten für Schiffe bei Landungen, Marine-Rdsch. 5 (1894) 225.

43 Diodonus, Historische Bibliothek, Deutsche Übersetzung von J.F. Wurm, Lib. 1, Band 1, Stuttgart 1827, p. 69.

44. DiRKsen E., Über Bleivergiftung an Bord an Kriegsschiffen, Marine-Rdsch. 5 (1894) 135 und 181.

45 Droвeк, Vom Wasservorkommen bis zur Schiffsversorgung, Mitt. dtsch. Ges. Verkehrsmed. 1962 H. 11, p. 2.

46 Elkinton J. R. und A. W. Winkler, Physiologic Effects of Drinking Undiluted SeaWater, War Med. 6 (1944) 24.1.

47 Engel S., Neuer Versuch über die Lage der nördlichen Gegenden von Asien und Amerika ..., Hauptst. 4 § 3, Bern 1777, p. 164.

48 Estenberg C., Nachrichten über den Gesundheitszustand der Mannschaften auf der «Nadesha » während der Reise um die Welt in den Jahren 1803, 1804, 1805 und 1806, in: A. J. v. Krusenstern, Reise um die Welt in den Jahren 1803, 1804, 1805 und 1806, Band 3, St. Petersburg 1812, p. 203 und 232.

49 Ferchl F., Chemisch-Pharmazeutisches Bio- und Bibliographikon, Mittenwald 1937, p. 155.

50 Fioravanti L., Della Fisica, Lib. 1, Cap. 95, Venedig 1603, p. 133.

51 Fonssagrives J.-B., Traité d'hygiène navale ... , Paris 1856.

51a Forget C. P., Médecine navale ..., Paris 1832.

52 Forster J. R., Observations faites pendant le second voyage de M. Cook dans l'hémisphère austral et autour du monde, Sect. 3, Band 5, Paris 1778, p. 50.

53 Fonster J. R., Physische Erdbeschreibung, Berlin 1783, p. 553.

54. Fraboulet A., L'historique des eaux de boisson à bord des navires, Thèse méd., Paris 1938.

55 Freycinet L. De, Notice sur l'histoire du voyage de l'Uranie, C. R. Acad. Sci. (Paris) $10(1840) 885$.

56 Freycinet L. De und Clément, Mémoires sur la distillation de l'eau de mer, et sur les avantages qui en résultent pour la navigation, Ann. Chim. Phys. 4 (1817) 225.

57 Friedel C., Die Krankheiten der Marine, Berlin 1866.

58 Friedmann S., Über Arzneikunde auf Kriegsschiffen, Erlangen 1850, p. 10.

59 Gardner F. T., Pedro Prat; Surgeon of the Spanish Conquest, Ann. Hist. Med. 3 (1941) 501.

60 Gehlen A. F., Über die Conservation des Wassers auf Seereisen, des Weines und anderer Flüssigkeiten, J. Chem. Physik 1 (1805) 645.

61 GÉrardin A., Altération, corruption et assainissement des rivières, Ann. Hyg. publ. (Paris) 43 (1875) 5.

62 Gilbertus Anglicus, Compendium medicinae, Lyon 1510, fol. 362 (De regimine transfretatium). 
63. Grass J., James Lind, M. D., Eighteenth-century Naval Medical Hygienist, J. Roy. Nav. Med. Serv. 35 (1949) 1 und 68.

64 Glatzel H., Die Situation des Schiffbrüchigen in ernährungsphysiologischer Sicht, Med. Klin 55 (1960) 1308.

65 Glauber J. R., Consolatio navigantium oder Trost der Seefahrenden, in: Glauberus concentratus oder Kern der Glauberischen Schriften ..., Leipzig-Breslau 1715, p. $501 \mathrm{ff}$.

66 Godfrey G., Miscellanea vere Utilia or Miscellanneous Experiments and Observations on Various Subjects $\left(2^{\circ}\right)$, London 1737.

67 Godlewski G., Le radeau de la «Méduse» et son chirurgien, Sem. Hop. 37 (1961) 3627.

68 Gonzales P. M., Tratado de las enfermedades de la gente de mar, en que se exponen sus causas, $y$ los medios de precaverlas, Madrid 1805.

69 Grundblatt S., Les chirurgiens de l'Hotel Dieu de Nantes sous l'Ancien Régime, Thèse méd., Nantes 1961.

70 GuYon L. B., Note sur les moyens de conserver l'eau dans les voyages de long cours et leur appréciation à la conservation des vins, Ann. Chim. 59 (1806) 96.

71 Hakluyt R., The Principal Navigations, Voyages, Traffiques and Discoveries of the English Nation, Extra Series, Glasgow Band 1-12, 1903-1905.

72 Hales S., Philosophical Experiments, Containing Usefull, and Necessary Instructions for Such as Undertake Long Voyages at Sea, London 1739.

73 Hales S., Account of Usefull Discovery to Distill Double the Quantity of Seawater by Blowing Showers of Air Through the Distilling Liquor, London 1755.

74. Hallbauer F. J. C. (Präses: C. F. Kaltschmied), De Nausea. Germ. die Seekrankheit adpellatur, Med. Diss., Jena 1765, p. 15.

75 Harlow C., Die Ernährung beim Sport, Berlin 1902, p. 59.

76 (HAuton), An Extract of a Letter From a Learned French Gentleman Concerning a Way of Making Sea-Water Sweet, Philosophical Trans. 1671, Nr. 67, p. 2048.

77 Hawkins R. Sir, Observations on a Voyage to the South Seas, siehe: Shaw, Abridgement of Mr. Boyle's Works, Band 3, London s. a., p. 220.

78 Henning F., Diätetisch-medizinisches Handbuch für Seeleute, Leipzig 1800.

79 Hervey G. R. und R. A. McCange, Effects of Carbohydrate and See Water on Metabolism of Men without Food or Sufficient Water, Proc. Roy. Soc. Med. 139 (1952) 527.

$79 a$ Hey G. A. (Präses: C. C. Schmiedel), De morbo ex navigatione oriundo, Med. Diss., Erlangen 1748, p. 13.

80 Hippokrates, CEuvres complètes, herausgegeben von E. Littré, Band 2, Paris 1840, p. 26f. Deutsche Übersetzung: Sämtliche Werke, herausgegeben von R. FucHS, Band 1, München 1895, p. $381 \mathrm{ff}$.

81 Huber, Über die Mittel zur Herstellung genußfähigen Wassers aus Meerwasser, Marine-Rdsch. 9 (1898) 1045, 1129, 1369, 1551, 1686.

82 Hudson A., A. Edward und A. Herbert, James Lind. His Contributions to Shipboard Sanitation, J. Hist. Med. 11 (1956) 1.

83 Hullu J., De Voeding op de Schepen der Oost-indische Compagnie, Bijdrag. Taal-, Land-en Volkenkunde Nederlaandsch-Indië 67 (1913) 541. 
84. HüsSEm B. H., Middel om het Water, welk tot dagelykschen drank voor het scheepvolk aan bord moet strekken, volkomen te zuiren, als het stinkend en bedorven is, Amsterdam 1799.

85 Inving G. J., Beschreibung der Methode des Dr. Irving, das Seewasser durch Distillieren süß zu machen, in: C. PHIPPs, Reise an den Nordpol, Deutsche Übersetzung von ENGEL, Bern 1772, p. $111 \mathrm{ff}$.

86 Keevil J. J., Leonard Gillespie, M. D., 1758-1842, Bull. Hist. Med. 28 (1954) 301 und 332 .

87 KeEvil J. J., Medicine and the Navy 1200-1900, Bände 1 und 2, Edinburgh/London 1957-1958.

88 Keraudren P.-F., Mémoire sur les causes des maladies des marins et sur les soins à prendre pour conserver leur santé dans les ports et à la mer, Paris 1817.

$88 a$ Keraudren P.-F., Expériences faites à Brest, Toulon et Rochefort sur l'eau de mer distillée employée en boisson et dans la préparation des aliments, Ann. maritim. colon. 1818, 12.

89 Kluge F., Etymologisches Wörterbuch der deutschen Sprache, $18^{\circ}$, herausgegeben von W. MitzKa, Berlin 1960.

90 Koeppe H., Reines Wasser, seine Giftwirkung und sein Vorkommen in der Natur, Dtsch. med. Wschr. 24. (1898) 624.

91 Krebel R., Geschichte und chronologische Übersicht der Gesammtliteratur des Skorbuts, St. Petersburg 1849.

92 Krebel R., Der Scorbut in geschichtlich-literarischer, pathologischer, prophylactischer und therapeutischer Beziehung, Leipzig 1862, p. $241 \mathrm{f}$.

93 La Billardiène J. J., Relation du voyage à la recherche de La Pérouse, fait per ordre de l'Assemblée constituante pendant les années 1791..., Band 1, Paris 1800 (an VIII), p. $55 \mathrm{f}$.

94. Laguna A., Compendium curationis praecautionisque morbi passim populariterque grassatis..., Straßburg 1542, letzte Seite.

95 La Pérouse J. F. DE, Voyage de la Pérouse autour du monde, Band 4, p. 237, Paris 1797.

96 LeFÈvne A., Recherches sur les causes de la colique sèche, Paris 1859.

97 Lefur J.-F., Etienne Chardon de Courcelles et le service de santé de la marine à Brest au XVIII ${ }^{e}$ siècle, Thèse med., Rennes 1959, p. $56 \mathrm{ff}$.

98 LeibBrand W. und A. Wettley, Der Wahnsinn, Freiburg/München 1961, p. 346 und 648.

99 Lery J. DE, Histoire d'un voyage faict en la Terre du Brésil, autrement dite Amerique, Cap. 4, Genf 1580, p. 32 f.

100 Leutmann J. G., Vulcanus famulans ..., Wittenberg 1735.

101 Leza G., True Account of the Events of the Voyage that the Captain Pedro Fernandez de Quiros Made to the Unknown Southern Lands, in: The Voyages of Pedro Fernandez de Quiros 1595-1606, Englische Übersetzung und herausgegeben von SIR C. Markham, Band 1, London 1904, p. 196, Band 2, p. 333.

102 Lind J., An Essay on the Most Effectual Means of Preserving the Health of Seamen in the Royal Navy, London 1757, $2^{\circ}$, London 1762. Deutsche Übersetzung Leipzig 1760.

103 Lind J., An Essay on Diseases Incidental to Europeans in Hot Climates, London 1768. 
104 Lippmann O. v., Die «Entsalzung des Meerwassers» bei Aristoteles, Chemiker-Ztg. 35 (1911) 629.

105 Lister M., A Way of Distilling Sweet and Fresh Water From Seawater by the Breath of Sea Plants Growing in It, Philosophical Trans. 1684, Nr. 156, p. 489.

106 Lloyd C. und J. L. S. Coulter, Medicine and the Navy 1200-1900, Band 3, Edinburgh/London 1961.

107 Lomonossow M. W., Gedanken vom Ursprunge der Eisberge im nordischen Meere, Abh. Schwed. Akad. Wiss. 25 (1763/66) 37. Deutsche Übersetzung von A. G. KästNER.

108 Lorgna, Sur la dulcification de l'eau de la mer, Ann. Chim. 12 (1792) 160.

109 Lorgna, Über die Versüßung des Meerwassers, Chem. Ann. 2 (1795) 321.

110 Lutrario A., La médecine navale au moyen âge et à l'époque moderne, Bull. Off. Internat. Hyg. Publ. 24 (1932) 1448.

111 Machine for the Purification of Sea Water, London Gazette 1684.

112 Mandelsloh J.A. von, Morgenländische Reyse Beschreibung, herausgegeben von A. Olearius, Schleswig 1658, p. 42.

113 Marsigli L. F. DE, Histoire physique de la mer, Amsterdam 1725, P. II, p. 25, 33, 41.

114 Matringly G., The Armada, Boston 1959. Deutsche Übersetzung: Die Armada, herausgegeben von G. Meyer-Clason, München 1960, p. 271.

115 McGance R. A., C. G. Ungley, J. W. L. Corsfill und E. M. Widdowson, The Hazards to Men in Ships Lost at Sea 1940-1944. Spec. Rep. Ser. Med. Res. Counc. (London) Nr. 291, London 1956, p. 20 ff. Ref.: Med. H. 51 (1956) 1842 und Lancet II (1956) 879.

116 MacDonald J. C., Outlines of Naval Hygiene, London 1881, p. 141.

117 Le médecin et la mer au temps de la Marine à voile, herausgegeben von J. SonOLET und P. Huard, Paris 1961 (Ausstellungsführer).

118 Menus K. F. H., Die hygienische Seite der Wasseraufbereitung an Bord, Hansa 99 (1962) 607.

119 Merleau-Ponty J.-J., Une page de l'histoire de la médecine navale: Amédée Lefèvre (1798-1869). Les médecins de la marine et la colique sèche, Thèse méd., Bordeaux 1895.

120 Mettler C. C., A History of Medicine, Philadelphia/Toronto 1947, p. 198.

121 Meyerhof M. und H. C. Rogge, Medizinisches aus Reisebeschreibungen nach Niederländisch Indien im 17. Jahrhundert, Janus 43 (1939) 92.

122 Minning H., Über Entwicklung der Hygiene und sanitären Ausrüstung auf Seeschiffen, Med. Mitt. Schering-Kahlbaum 2 (1930) 95.

123 Monson W. Sir, Naval Tracts, herausgegeben von M. OppenheIm, Band 1, London 1902, p. 226 ff.; Band 5, London 1914, p. 179 f.

124. Moryson F., Itinerary, Part 1, B. 3, Cap. 1, London 1617, p. 208.

125 Müller G., Probleme einer bakteriologisch einwandfreien Trinkwasserversorgung auf kleineren Schiffen, Städtehygiene 12 (1961) 113.

126 Niebuhr C., Reisebeschreibung nach Arabien und anderen umliegenden Ländern, Band 1, Kopenhagen 1774, p. 12.

127 Nоснт B., Vorlesungen für Schiffsärzte, Leipzig 1906, Kap. 13, p. $223 \mathrm{ff}$.

128 OenLer R., Zur Schädlichkeit des destillierten Wassers, Münch. med. Wschr. 59 (1912) 2729.

129 Oesterlen F., Handbuch der Hygiene, Tübingen 1851, p. 453. 
130 Opıтz K., Avicenna. Das Lehrgedicht über die Heilkunde, aus dem Arabischen übersetzt, Quell. Stud. Gesch. Med. Naturw. 7 (1940) 150.

131 Отто J. F. W., Abriß einer Naturgeschichte des Meeres, Berlin 1792.

132 Percival R., An Account of the Cape of Good Hope, London 1804. Beschreibung des Vorgebirges der Guten Hoffnung, Deutsche Übersetzung von T. F. EhrmanN, in: Bibliothek der neuesten und wichtigsten Reisebeschreibungen, Band 18, Weimar 1805, p. 45.

133 Pezzi G., Sui progressi dell'igiene e della medicina navale nei secoli XIX e XX, Riv. Storia Sci. Med. Nat. 44 (1953) 194.

134. Pezzi G., Medicina navale del 700, Ann. Med. Nav. Trop. 52 (1947) 427.

135 Pezzi G., Una rara opera di terapia spagirica del sec. XVII al uso di naviganti, Ann. Med. Nav. Trop. 53 (1948) 333.

136 Pezzi G., La medicina navale nella storia, in: Medicina navale, herausgegeben von A. Mirra, Padua 1961, p. $1 \mathrm{ff}$.

137 Phipps C., Voyage on the North Pole, London. Deutsche Übersetzung: Reise an den Nordpol, herausgegeben von EngeL, Bern 1772, p. 11.

138 Phipson T. L., La force catalytique aux études sur les phénomènes de contact, Haarlem 1858.

139 Pictorius G., Raiß-Buechlin. Ordnung wie sich zu halten, so einer raisen will in weite und onerfarne Land ..., Straßburg 1557.

140 Plinius, Historia naturalis, herausgegeben von W. R. S. Jones, Band 1, London Cambridge, 1934, Lib. 2, p. 104 und 234.

141 Poissonnier-Desperriènes A., Traité des maladies des gens de mer, Paris 1767.

142 Pop G. F., De geneeskunde bij het Nederlandsche Zeewezen, Geneesk. Tijdsch. Zeemagt 1866-1868 und herausgegeben von L. S. v. Römer, Weltevreden 1922.

143 Pringle J. Sir, A Discourse upon Some Late Improvements of the Means for Preserving the Health of Mariners, London 1776. Deutsche Übersetzung von Wichmann: Über einige neuere Verbesserungen der Mittel, die Gesundheit der Seeleute zu erhalten, Göttingen 1777 , p. 59.

144 Renner P., Das Kriegstagebuch Herzog Philipps von Cleve, Phil. Inaug. Diss., Heidelberg 1960 , p. $148 \mathrm{ff}$.

145 Reyer S., Experimentum novum, quo aquae marinae dulcedo examinata describitur, Kiel 1679.

146 Richter A. P. F., Consolatio Navigantium, Marine-Rdsch. 23 (1912) 1285.

147 RiEgel W., Heizung, Beleuchtung, Wasserversorgung, Bade- und Wascheinrichtungen, Eisbereitung und Kälteerzeugung, Beseitigung von Abfallstoffen, Ungeziefervertilgung, in: Handbuch der Gesundheitspflege an Bord von Kriegsschiffen, herausgegeben von M.zur Verth, E. Bentmann, E. Dirksen und R. Ruge, Band 1, Jena 1914, p. $547 \mathrm{ff}$.

148 Rocher und Peyre, Description et figure d'un appareil pour la déstillation de l'eau de mer à bord des vaisseaux, C. R. Acad. Sci. (Paris) 13 (1841) 206.

149 Roddis L. H., A Short History of Nautical Medicine, New York-London 1941, und Ann. Med. Hist. 3 (1941) ser. 3, p. 203, 326 und 418.

150 Roddis L. H., James Lind, Founder of Nautical Medicine, New York 1950.

151 Rouppe L., De Morbis Navigantium, Leiden 1764, p. 317. 
152 Ruge R., Schiffsmedizinisches im 16. und 17.Jahrhundert, Marine-Rdsch. 11 (1900) 1244.

153 Ruge R., Fortschritte in der Schiffshygiene während des 19. Jahrhunderts, MarineRdsch. 24 (1913) 576.

154. Ruge R., Geschichte der Schiffshygiene, in: Handbuch der Gesundheitspflege an Bord von Kriegsschiffen, herausgegeben von M. zur Verth, E. Bentmann, E. Dirksen und R. Ruge, Band 1, Jena 1914, p. $26 \mathrm{ff}$.

155 Russel R., De tabe glandulari seu de usu aquae marinae et morbis glandularum... dissertatio, Oxford und London 1750. p. 77 und 232.

156 SaAr J. J., Ost-Indianische fünfzehnjährige Kriegsdienste, Nürnberg 1662. Reise nach Java, Banda, Ceylon und Persien 1644-1660, in: Reisebeschreibung deutscher Beamter und Kriegsleute im Dienste der Niederländischen West- und Ostindischen Kompagnien, herausgegeben von S. P. L'Honoré NABER, Band 6, Den Haag 1930, p. 16.

157 SAge B. G., Expériences qui font connaître qu'on ne peut admettre l'innocuité de l'eau de mer distillée parce qu'elle contient toujours du gaz alcalin oléaginé neptunien produit par la putréfaction des êtres organisés marins, Paris 1817.

158 Samhaber E., Knaurs Geschichte der Entdeckungsreisen, München 1955, p. $330 \mathrm{ff}$.

159 Savigny J. B., Observations sur les effets de la faim et de la soif éprouvées après le naufrage de la frégate du roi «La Méduse» en 1816, Thèse méd., Paris 1818.

160 Schade W., Medizinisch-technische Probleme des Schiffbruchs, Bremer Ärztebl. 13 (1960) 4.

161 Schadewaldt H., Der Schiffsarzt, Ciba-Z. (Wehr) 7 (1955) $2502 \mathrm{ff}$.

162 Schadewaldt H., Die Bedeutung der Schiffsmedizin für die gesamte Heilkunde, Med.Welt 1961, Nr. 8, p. 398, 1961, Nr. 9, p. 442.

163 Schadewaldt H., Geschichte der Schiffahrtsmedizin und der Marinepharmazie, in: Wehrdienst und Gesundheit (Sonderband), 1963 (im Druck).

164. Schoute D., De geneeskunde in den dienst der Oost-Indische Compagnie in Nederlandsch-Indië, Amsterdam 1929.

165 Sсотт H. H., A History of Tropical Medicine, Band 1, London s.a., p. 35.

166 SÉGond A., Essai sur la névralgie du grand sympathique, maladie connue sous le nom de colique végétable..., Paris 1837.

167 Seume J. F., Mein Leben, Leipzig 1813, p. 131.

168 Smollet T., The Adventures of Roderick Random. Deutsche Übersetzung von W. C. S. Mylius. Die Abenteuer von Roderick Random, Band 1, Berlin 1790, p. 515.

168 a Sprengel K., Versuch einer progmatischen Geschichte der Arzneikunde, Band 2, Halle 1828, p. 414.

169 StaAcK H. H., Welche Forderungen sind von seiten der Hygiene an eine einwandfreie Trinkwasseranlage an Bord zu stellen? Wehrmed. Mitt. 1961, H. 12, p. 177.

170 StaAcK H. H., Hygienische Aspekte der Trinkwasserversorgung auf Schiffen und Vorschläge zur Verbesserung und Überwachung entsprechender Anlagen an Bord, Mitt. dtsch. Ges. Verkehrsmed. 1962, H. 11, p. 12.

171 Steffenhagen C., Wasser-Versorgung an Bord von Schiffen, Mitt. dtsch. Ges. Verkehrsmed. 1962, H. 11, p. 45.

172 Steward C. P. und D. Guthrie, Lind's Treatise on Scurvy: A Bicentenary Volume, Edinburgh 1953, p. 145. 
173 StipriaAn-Luiscius A. van, Über die Reinigung verdorbenen faulen Wassers, J. Chemie Physik 1 (1806) 621.

174. Strandes J., Die Portugiesenzeit von Deutsch- und Englisch-Ostafrika, Berlin 1899, p. 31.

175 Tidow R., Ärztliche Fragen bei Seenot, Wehrmed. Mitt. 1960, H. 2, p. 17, und 3, p. 37.

176 Torquemada F. J., La monarquia indiana, Clavijò 1613, in: The Voyages of Pedro Fernandez de Quiros 1595-1606. Englische Übersetzung und herausgegeben von Sir C. Markham, Band 2, London 1904, p. 413.

177 Trotter T., Medicina nautica, London 1797-1803.

178 Villagrazia M. De, Storia di medicamenti marini, Atti Accad. Storia Arte sanit. 20 (1954) ser. 2, Nr. 3.

179 Volhard F. und E. Schütte, Über die Verträglichkeit von Meerwasser, Dtsch. med. Wschr. 75 (1950) 1425.

180 Vrolingh A. L., Der matrosen gesontheyt, Amsterdam 1666, p. 45.

181 WaIz J., Untersuchung der Ursache, warum Wasser im atlantischen Meere allezeit in das mittelländische Meer durch die Enge bey Gibraltar hineinströmt, Abh. Schwed. Akad.Wiss. 17 (1757) 28, deutsche Ausgabe von A. G. Kästner.

182 Walcot W., An Answer to Mr. Fitzgerald's State of the Case Concerning the Patent of Making Salt-Water Fresh, London 1695.

183 Walcot W., A Treatise Concerning the Exercise of W.W. His Art of Making SaltWater Fresh ..., London 1697.

184. Watson R., An Account of Mr. Appleby's Process to Make Seawater Fresh, Philosophical Trans. $48(1753 / 54) 69$.

185 Wilson J., A Manual of Naval Hygiene, London 1879.

186 Wттн O. H., Die Gesundheitspflege auf Seeschiffen für Gebildete aller Stände, namentlich für Schiffsoffiziere und Auswanderer ..., Bremerhaven 1858.

187 WurfFbaIN J.S., Vierzehnjährige Ost-Indianische Kriegs- und Oberkauffmannsdienste. Nürnberg 1686. Reise nach den Molukken und Vorderindien 1632-1646, in: Reisebeschreibungen von deutschen Beamten und Kriegsleuten im Dienst der Niederländischen West- und Ost-Indischen Kompagnie 1602-1797, Bände 1-2, Den Haag 1931, herausgegeben von R. P. MEYJES.

188 ZовеL M., Les naturalistes voyageurs français et les grands voyages maritimes du XVIII et $X I X^{e}$ siècles, Thèse méd., Paris 1961. 